\title{
Oxidative Cyclization Approach to Benzimidazole Libraries
}

Eric P. Arnold, Prolay K. Mondal, and Daniel C. Schmitt*

Pfizer Worldwide R\&D, Eastern Point Road, Groton, Connecticut 06340, United States

Supporting Information

\section{Table of Contents}

General Information.

General Procedures . . $\mathrm{S} 3$

Spectroscopic Data for Scheme 2

Spectroscopic Data for Scheme 3

Experimental Procedures for Scheme 4

Experimental Procedures for Scheme 5 
General Information: All reagents were obtained from commercial suppliers and used without further purification, unless otherwise noted. All solvents used were purchased anhydrous and transferred by nitrogen-purged syringe. Silica gel chromatography was performed using medium pressure Biotage or ISCO systems employing columns pre-packaged by various commercial vendors including Biotage and ISCO. ${ }^{1} \mathrm{H}$ and ${ }^{13} \mathrm{C}$ NMR characterization data were collected at $300 \mathrm{~K}$ on a Bruker AS- 400 spectrometer operating at 400 and $100 \mathrm{MHz}$ (respectively) with chemical shifts reported in parts per million relative to $\mathrm{CD}_{3} \mathrm{OD}\left({ }^{1} \mathrm{H}\right.$ NMR: $3.31 \mathrm{ppm} ;{ }^{13} \mathrm{C}$ NMR: $\left.49.0 \mathrm{ppm}\right)$. LC-MS were acquired using a Waters Acquity UPLC equipped with a Waters Acquity HSS T3 column, water $/ \mathrm{MeCN}$ gradient and $0.1 \% \mathrm{v} / \mathrm{v}$ formic acid as modifier. 
General Library Procedure A: To a plate of 2-dram vials with septum caps containing the anilines $(0.40 \mathrm{mmol}, 1.0$ equiv) was added THF $(2.5 \mathrm{~mL}, 0.16 \mathrm{M})$ followed by LiHMDS $(0.40 \mathrm{~mL}, 1 \mathrm{M}$ solution in THF, 1.0 equiv). The resulting solutions were stirred at $23{ }^{\circ} \mathrm{C}$ for 20 minutes. Benzonitrile ( $41 \mu \mathrm{L}, 0.40$ mmol, 1.0 equiv) was added via syringe and the reactions were stirred at $23{ }^{\circ} \mathrm{C}$ for $17 \mathrm{~h}$. Solvent was then removed via genevac to provide the crude amidine intermediates. DMSO $(2.5 \mathrm{~mL}, 0.16 \mathrm{M})$ and $\mathrm{Cu}(\mathrm{OAc})_{2}$ (145 mg, $0.80 \mathrm{mmol}, 2.0$ equiv) were added followed by glacial acetic acid ( $0.46 \mathrm{~mL}, 8 \mathrm{mmol}, 20$ equiv). Without capping the vials, they were placed in a preheated $110{ }^{\circ} \mathrm{C}$ plate and stirred for $17 \mathrm{~h}$ open to the atmosphere. The vials were cooled and poured into $\mathrm{Na}_{2} \mathrm{CO}_{3}(20 \mathrm{~mL}$, saturated aqueous solution $)$. The mixtures were extracted with EtOAc $(2 \mathrm{X} 5 \mathrm{~mL})$ and filtered through $\mathrm{Na}_{2} \mathrm{SO}_{4}$ plugs. Solvent was removed via genevac. The resulting crude benzimidazoles were dissolved in $1 \mathrm{~mL}$ DMSO and submitted for high-

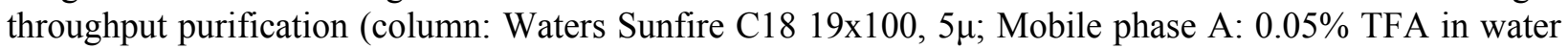
$(\mathrm{v} / \mathrm{v})$; Mobile phase B: $0.05 \%$ TFA in acetonitrile $(\mathrm{v} / \mathrm{v})$.

\section{General Library Procedure B:}

Imidoyl triflate stock solution: To a dry flask under $\mathrm{N} 2$ atmosphere was added $\mathrm{N}$-methyl benzamide (1.0 equiv per reaction) followed by $\mathrm{CH}_{2} \mathrm{Cl}_{2}(0.4 \mathrm{M})$ and 2,6-lutidine (2.2 equiv). The flask was cooled to $0{ }^{\circ} \mathrm{C}$ and triflic anhydride (1.1 equiv per reaction) was added dropwise. The solution was allowed to warm to $23{ }^{\circ} \mathrm{C}$ over $2.5 \mathrm{~h}$.

Amidine formation / oxidative cyclization: To a plate of 2-dram vials with septum caps containing the anilines (1.2 equiv) was added $\mathrm{CH}_{2} \mathrm{Cl}_{2}(0.8 \mathrm{M})$ followed by the imidoyl triflate solution (1.0 equiv per vial). Vials were stirred at $23{ }^{\circ} \mathrm{C}$ for $15 \mathrm{~h}$. Reaction mixtures were concentrated under a stream of $\mathrm{N}_{2}$, then partitioned between water and EtOAc (1:1). The organic phases were separated and the aqueous phases were re-extracted with EtOAc. The combined organic phases were filtered through $\mathrm{Na}_{2} \mathrm{SO}_{4}$ plugs into 2 dram vials and concentrated under a stream of $\mathrm{N}_{2}$. To the resulting crude amidines was added $\mathrm{MeCN}(0.25$ $\mathrm{M})$ and $\mathrm{PhI}(\mathrm{OAc})_{2}(2.0$ equiv per vial). The vials were sealed with pressure-release caps and heated to 100

${ }^{\circ} \mathrm{C}$ for $2 \mathrm{~h}$. The vials were cooled and concentrated under a stream of $\mathrm{N}_{2}$. The resulting residues were diluted with $\mathrm{H}_{2} \mathrm{O}$, extracted with EtOAc (2x), and filtered through $\mathrm{Na}_{2} \mathrm{SO}_{4}$ plugs. Solvent was removed under a stream of $\mathrm{N}_{2}$. The resulting crude benzimidazoles were dissolved in $1 \mathrm{~mL}$ DMSO and submitted

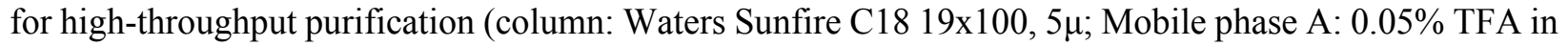
water (v/v); Mobile phase B: $0.05 \%$ TFA in acetonitrile (v/v). 


\section{Spectroscopic Data for Scheme 2}

Compounds were prepared in parallel format via General Library Procedure A.

Compound $\mathbf{2 f}$ and $\mathbf{2 i}$ has been fully characterized previously and NMR spectra were matched to reported values:

2f) Huang, J.; He, Y.; Wang, Y.; Zhu, Q. Chem. Eur. J. 2012, 18, 13964-13967.

2i) Kim, J.; Kim J.; Lee, H.; Lee, B. M.; Kim, B. H. Tetrahedron 2011, 67, 8027-8033.

\section{4-methoxy-2-phenyl-1H-benzo[d]imidazole (2a)}

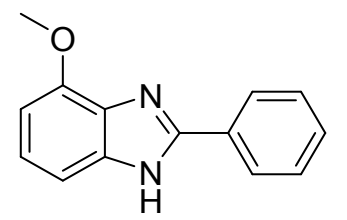

$\underline{{ }^{1} \text { H NMR }}\left(400 \mathrm{MHz}\right.$, METHANOL- $\left.d_{4}\right) \delta 8.15(\mathrm{~d}, J=7.3 \mathrm{~Hz}, 2 \mathrm{H}), 7.69-7.80(\mathrm{~m}, 3 \mathrm{H}), 7.54(\mathrm{t}, J=8.2 \mathrm{~Hz}$, $1 \mathrm{H}), 7.37(\mathrm{~d}, J=7.8 \mathrm{~Hz}, 1 \mathrm{H}), 7.13(\mathrm{~d}, J=7.8 \mathrm{~Hz}, 1 \mathrm{H}), 4.11(\mathrm{~s}, 3 \mathrm{H}) \mathrm{ppm}$.

${ }^{13}$ C NMR $\left(101 \mathrm{MHz}\right.$, METHANOL- $\left.d_{4}\right) \delta 149.26,147.72,133.43,133.17,129.59,129.55,127.72$, $127.37,123.16,106.27,105.41,55.36 \mathrm{ppm}$.

$\underline{\text { LC-MS }}$ (ESI) Calcd. for $\mathrm{C}_{14} \mathrm{H}_{12} \mathrm{~N}_{2} \mathrm{O}(\mathrm{M}+\mathrm{H}): 225.1$, Found: 225.3 

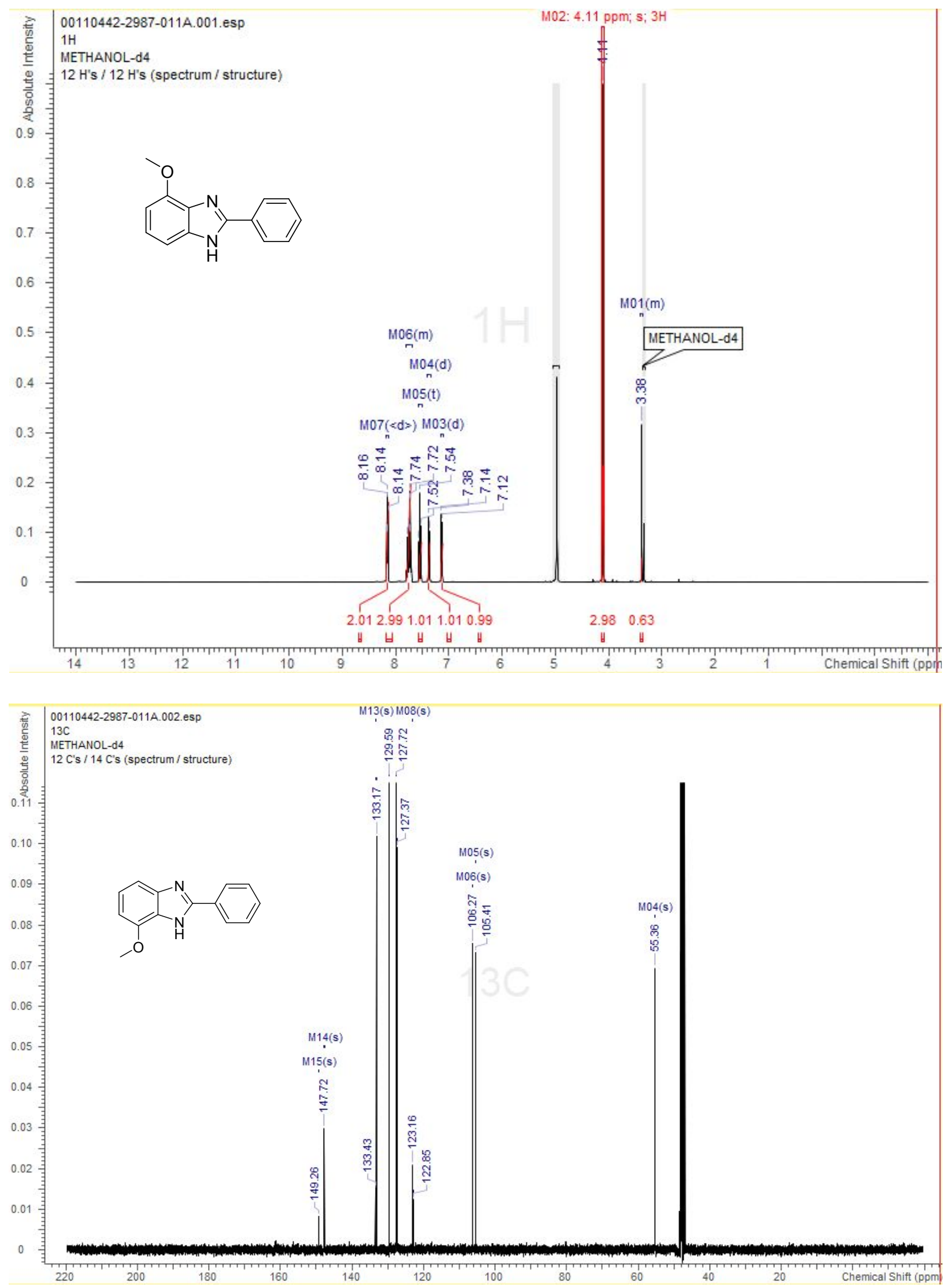


\section{4-fluoro-2-phenyl-1H-benzo[d]imidazole (2b)}

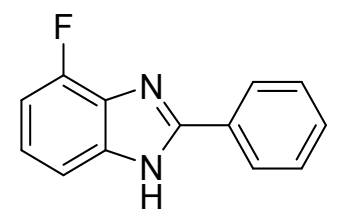

${ }^{1}$ H NMR (400 MHz, METHANOL- $\left.d_{4}\right) \delta 8.12$ - $8.22(\mathrm{~m}, 2 \mathrm{H}), 7.66-7.78(\mathrm{~m}, 3 \mathrm{H}), 7.59(\mathrm{~d}, J=7.9 \mathrm{~Hz}, 1 \mathrm{H})$, 7.50 (br d, $J=4.7 \mathrm{~Hz}, 1 \mathrm{H}), 7.30$ (t, $J=9.2 \mathrm{~Hz}, 1 \mathrm{H}) \mathrm{ppm}$.

${ }^{13}$ C NMR $\left(101 \mathrm{MHz}\right.$, METHANOL- $\left.d_{4}\right) \delta 151.70,149.21,136.36,132.60,129.43,127.53,125.79$, $124.13,110.18,110.01,109.47 \mathrm{ppm}$.

LC-MS (ESI) Calcd. for $\mathrm{C}_{13} \mathrm{H}_{9} \mathrm{FN}_{2}(\mathrm{M}+\mathrm{H})$ : 213.1, Found: 213.2 

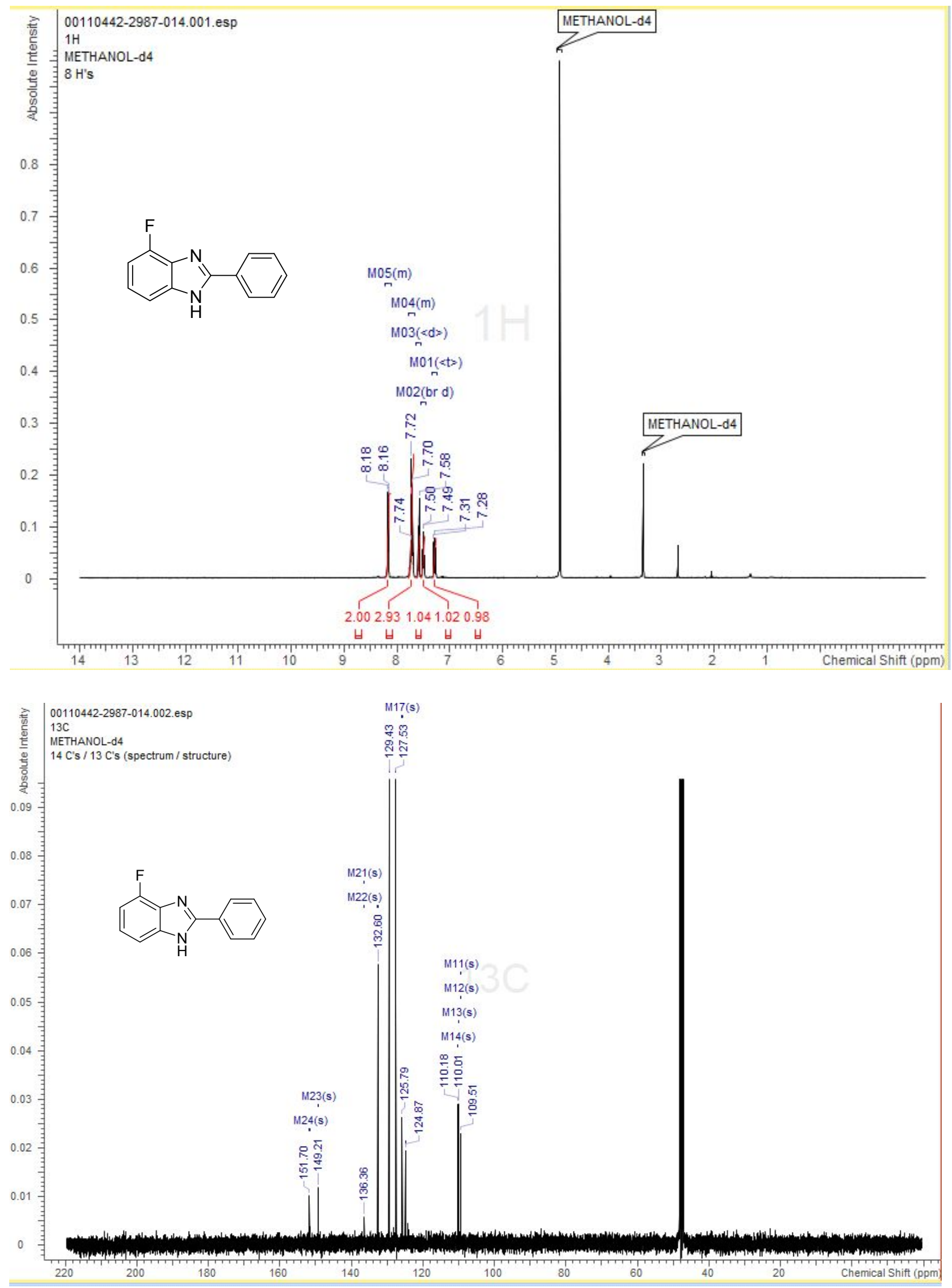


\section{5,7-dimethyl-2-phenyl-1H-benzo[d]imidazole (2e)}

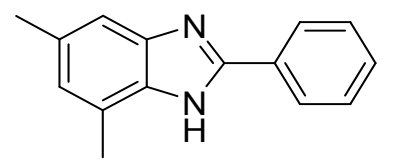

$\underline{\text { 1H NMR }}\left(400 \mathrm{MHz}\right.$, METHANOL- $\left.d_{4}\right) \delta 8.14$ - $8.20(\mathrm{~m}, 2 \mathrm{H}), 7.70$ - $7.81(\mathrm{~m}, 3 \mathrm{H}), 7.42(\mathrm{~s}, 1 \mathrm{H}), 7.25(\mathrm{~s}$, $1 \mathrm{H}), 2.68(\mathrm{~s}, 3 \mathrm{H}), 2.53(\mathrm{~s}, 3 \mathrm{H}) \mathrm{ppm}$.

${ }_{13}^{13}$ NMR $\left(126 \mathrm{MHz}\right.$, METHANOL- $\left.d_{4}\right) \delta 149.25,136.82,132.86,132.62,130.26,129.50,128.16$, 127.55, 124.24, 123.67, 110.48, 20.24, $15.44 \mathrm{ppm}$.

$\underline{\text { LC-MS }}$ (ESI) Calcd. for $\mathrm{C}_{15} \mathrm{H}_{14} \mathrm{~N}_{2}(\mathrm{M}+\mathrm{H}): 223.1$, Found: 223.2 


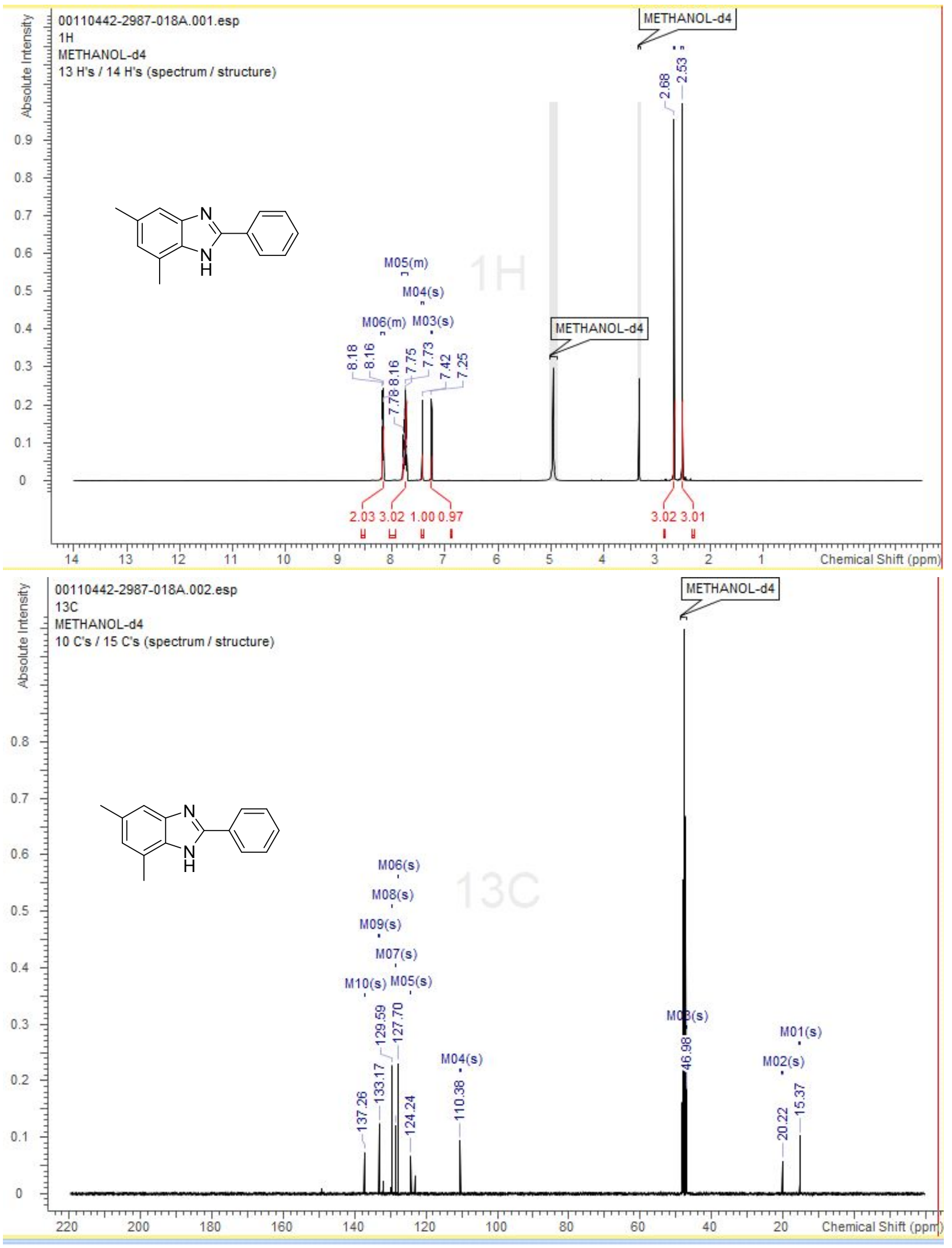




\section{5-(methylsulfonyl)-2-phenyl-1H-benzo[d]imidazole (2g)}

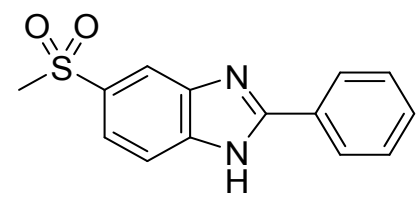

${ }^{1}$ H NMR $\left(400 \mathrm{MHz}\right.$, METHANOL- $\left.d_{4}\right) \delta 8.27(\mathrm{~d}, J=1.6 \mathrm{~Hz}, 1 \mathrm{H}), 8.16-8.14(\mathrm{~m}, 2 \mathrm{H}), 7.94(\mathrm{dd}, J=1.6$ $\mathrm{Hz}, 8.6 \mathrm{~Hz}, 1 \mathrm{H}), 7.87$ (d, $J=8.4 \mathrm{~Hz}, 1 \mathrm{H}), 7.65-7.63$ (m, 3H), 3.19 (s, 3H) ppm.

${ }^{13} \mathbf{C}$ NMR $\left(101 \mathrm{MHz}\right.$, METHANOL- $\left.d_{4}\right) \delta 152.6,150.5,141.9,137.4,133.2,130.7,129.0,128.6,123.8$, 116.6, 116.4, $45.1 \mathrm{ppm}$.

LC-MS (ESI) Calcd. for $\mathrm{C}_{14} \mathrm{H}_{12} \mathrm{~N}_{2} \mathrm{O}_{2} \mathrm{~S}(\mathrm{M}+\mathrm{H}): 273.1$, Found: 273.3. 

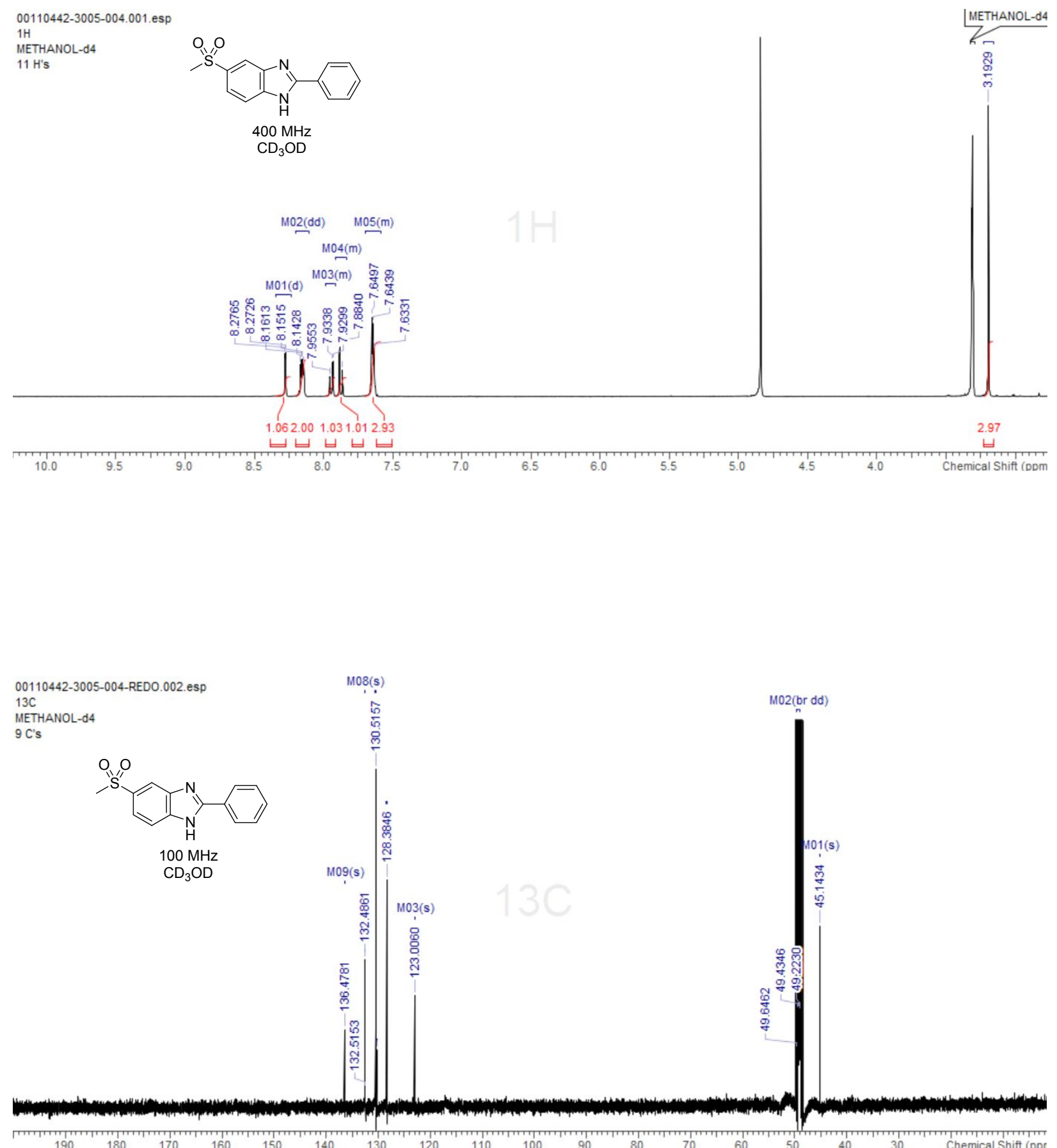


\section{1-(2-phenyl-1H-benzo[d]imidazol-7-yl)pyridin-2(1H)-one (2h)}

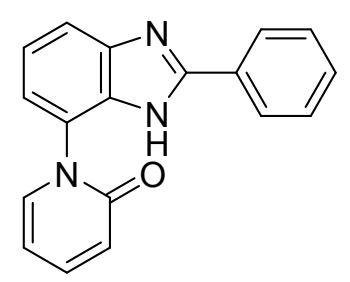

$\underline{{ }^{1} \text { H NMR }}\left(400 \mathrm{MHz}\right.$, METHANOL- $\left.d_{4}\right) \delta 8.06-8.17(\mathrm{~m}, 2 \mathrm{H}), 7.87(\mathrm{~d}, J=8.20 \mathrm{~Hz}, 1 \mathrm{H}), 7.60-7.77(\mathrm{~m}$, $5 \mathrm{H}), 7.47(\mathrm{~d}, J=7.02 \mathrm{~Hz}, 1 \mathrm{H}), 6.75(\mathrm{~d}, J=9.28 \mathrm{~Hz}, 1 \mathrm{H}), 6.61-6.59(\mathrm{~m}, 1 \mathrm{H}) \mathrm{ppm}$.

${ }_{13}^{13}$ NMR $\left(101 \mathrm{MHz}\right.$, METHANOL- $\left.d_{4}\right) \delta 164.7,153.5,143.3,140.3,137.1,134.0,132.7,130.9,129.6$, $129.2,126.9,126.7,124.5,122.1,116.2,109.2 \mathrm{ppm}$.

$\underline{\text { LC-MS }}$ (ESI) Calcd. for $\mathrm{C}_{18} \mathrm{H}_{14} \mathrm{~N}_{3} \mathrm{O}(\mathrm{M}+\mathrm{H})$ : 288.3, Found: 288.4 
00110442-3005-022.3.fid

NMR System BNMR_311-1 400 BH013110

Pfizer Confidential

proton DMSO \{C:|Bruker|TopSpin3.0\} arnoldep 21

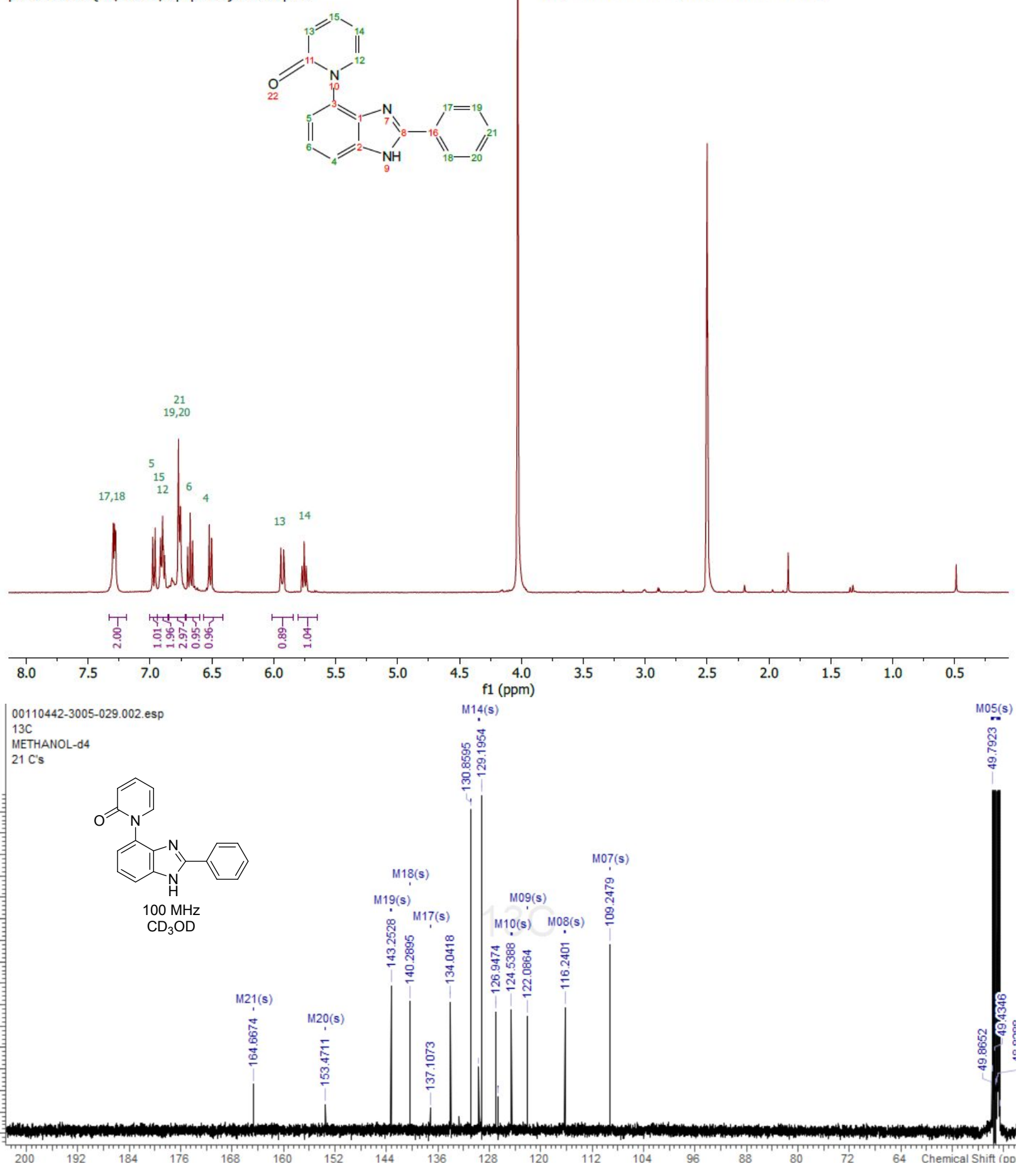

'HNMR (400 MHz DMSO $\left.d_{6}\right) 87.33-7.19(\mathrm{~m}, 2 \mathrm{H}), 6.97(\mathrm{dd}, J=82,0.9 \mathrm{~Hz}, 1 \mathrm{H}), 6.94-6.85$ $(\mathrm{m}, 2 \mathrm{H}), 6.76$ (dd, $J=5.0,1.9 \mathrm{~Hz}, 3 \mathrm{H}), 6.68(\mathrm{t}, J=7.9 \mathrm{~Hz}, 1 \mathrm{H}), 6.51(\mathrm{dd}, J=7.7,0.9 \mathrm{~Hz}, 1 \mathrm{H})$, $5.93(\mathrm{dd}, J=9.9,1.3 \mathrm{~Hz}, 1 \mathrm{H}), 5.75(\mathrm{td}, J=6.7,1.3 \mathrm{~Hz}, 1 \mathrm{H})$. 


\section{6-methoxy-2-phenyl-1H-benzo[d]imidazole (2j)}

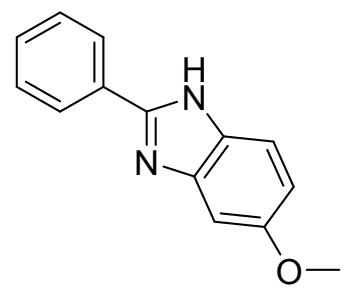

$\underline{{ }^{1} \mathbf{H} \text { NMR }}\left(400 \mathrm{MHz}\right.$, METHANOL- $\left.d_{4}\right) \delta 7.95-8.00(\mathrm{~m}, 2 \mathrm{H}), 7.54-7.66(\mathrm{~m}, 4 \mathrm{H}), 7.13(\mathrm{~d}, J=2.3 \mathrm{~Hz}, 1 \mathrm{H})$, $7.10(\mathrm{~s}, 1 \mathrm{H}), 3.80(\mathrm{~s}, 3 \mathrm{H}) \mathrm{ppm}$.

${ }^{13}$ C NMR $\left(101 \mathrm{MHz}\right.$, METHANOL- $\left.d_{4}\right) \delta 159.36,148.61,133.24,132.79,129.72,127.24,125.88$, $122.78,116.54,114.30,95.60,55.14 \mathrm{ppm}$.

$\underline{\text { LC-MS }}$ (ESI) Calcd. for $\mathrm{C}_{14} \mathrm{H}_{12} \mathrm{~N}_{2} \mathrm{O}(\mathrm{M}+\mathrm{H}): 224.09$, Found: 224.3 


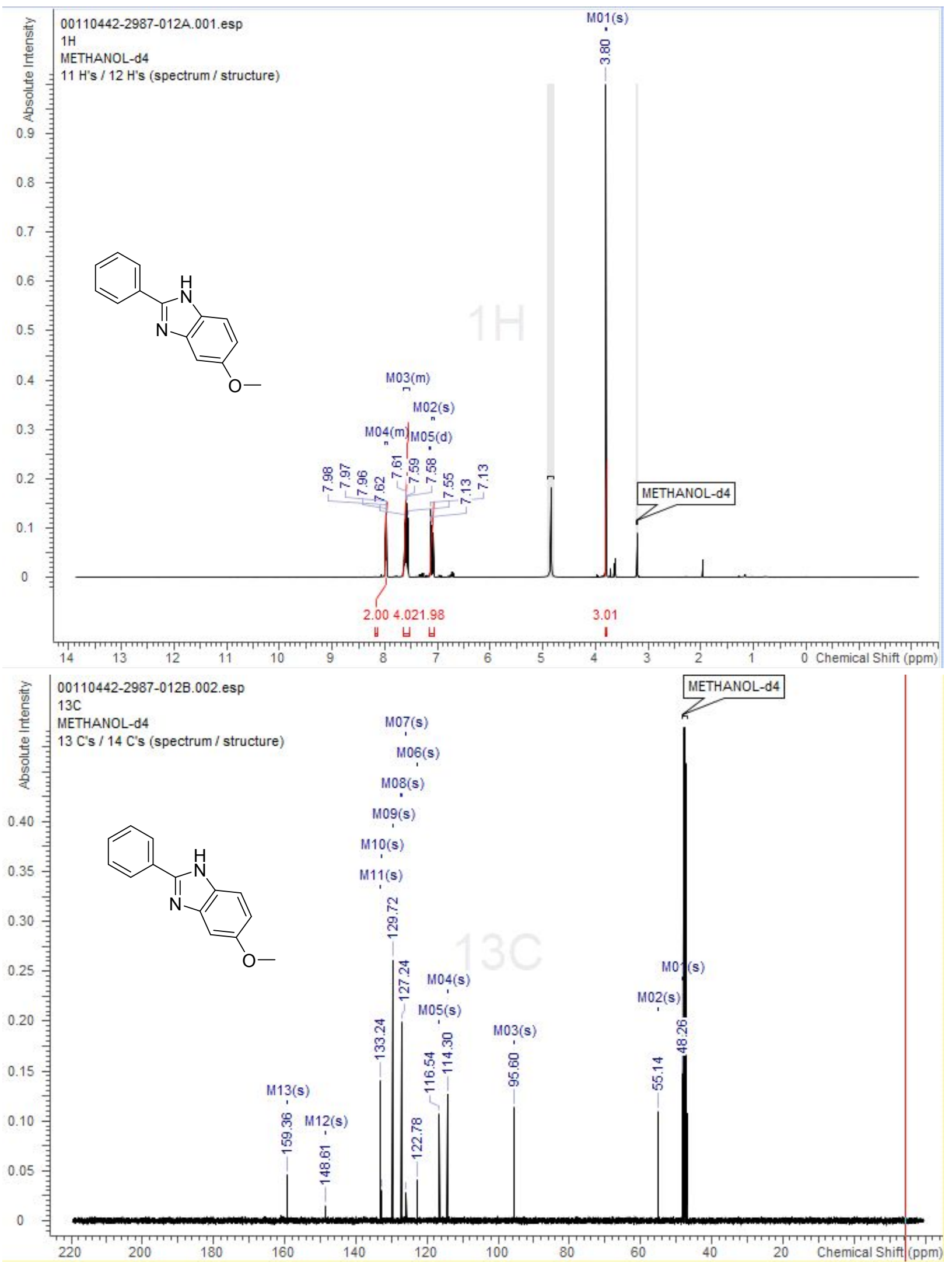




\section{6-methyl-2-phenyl-1H-benzo[d]imidazole (2k)}

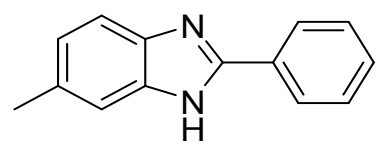

${ }^{1}$ H NMR $(400 \mathrm{MHz}$, METHANOL-d 4$): \delta 8.04-7.98(\mathrm{~m}, 2 \mathrm{H}), 7.68-7.56(\mathrm{~m}, 4 \mathrm{H}), 7.50(\mathrm{~s}, 1 \mathrm{H}), 7.34(\mathrm{dd}$, $J=8.6,0.8 \mathrm{~Hz}, 1 \mathrm{H}), 2.45$ (s, 3H) ppm.

13. NMR $\left(101 \mathrm{MHz}\right.$, METHANOL- $\left.d_{4}\right) \delta 149.19,137.26,133.32,132.24,130.07,129.72,127.89$, $127.42,123.05,113.16,113.05,20.29 \mathrm{ppm}$.

$\underline{\text { LC-MS }}$ (ESI) Calcd. for $\mathrm{C}_{14} \mathrm{H}_{12} \mathrm{~N}_{2}(\mathrm{M}+\mathrm{H}): 209.1$, Found: 209.2 


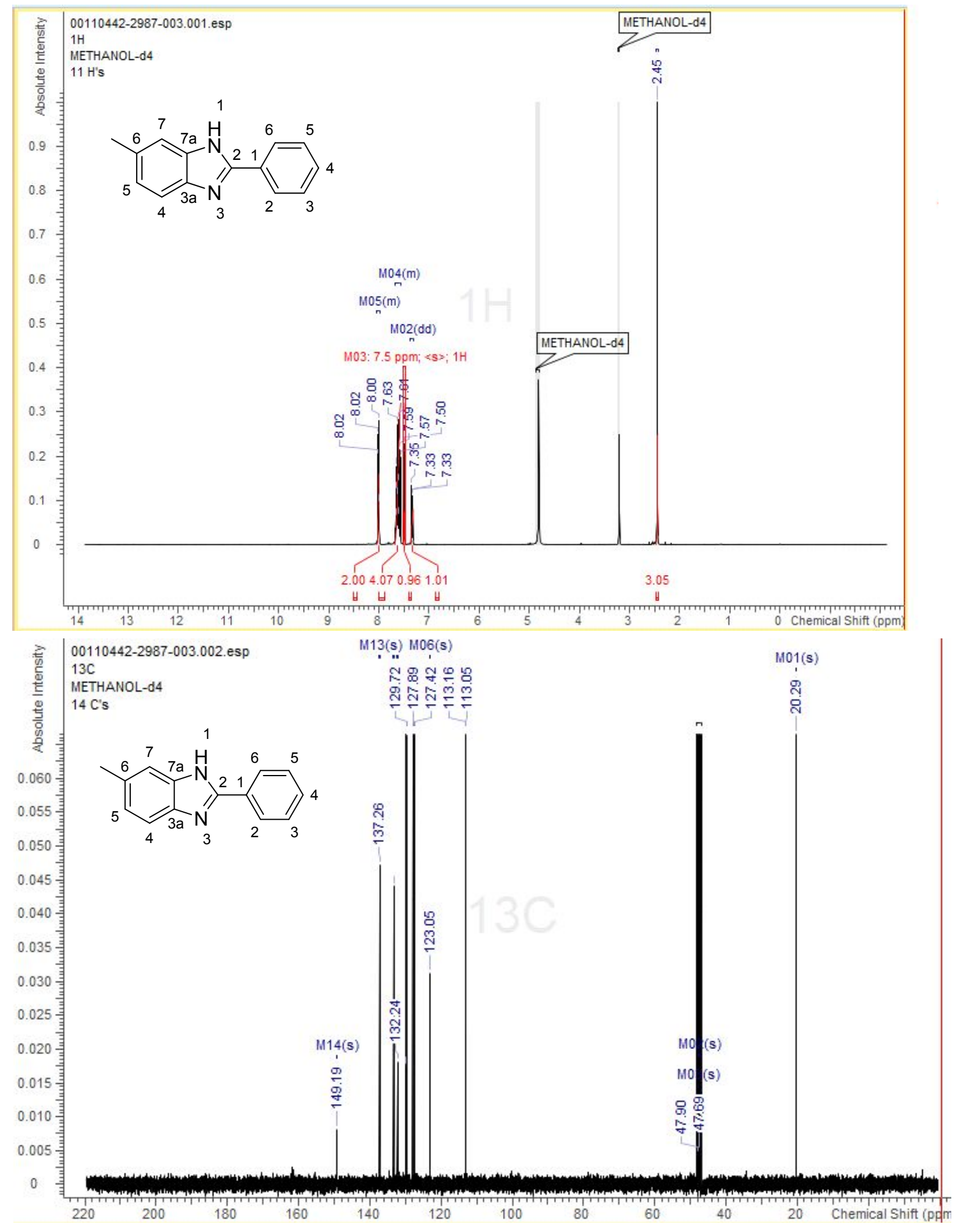


tert-butyl methyl((2-phenyl-1H-benzo[d]imidazol-6-yl)methyl)carbamate (2l)

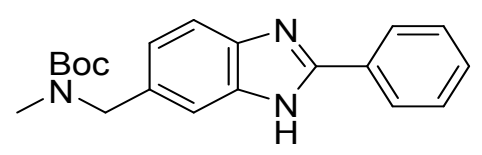

1H NMR $\left(400 \mathrm{MHz}\right.$, METHANOL- $\left.d_{4}\right) \delta 8.13(\mathrm{br} \mathrm{d}, J=7.41 \mathrm{~Hz}, 2 \mathrm{H}), 7.70$ - 7.90 (m, $\left.4 \mathrm{H}\right), 7.68$ (br s, 1 H), $7.52(\mathrm{~d}, J=8.20 \mathrm{~Hz}, 1 \mathrm{H}), 4.64(\mathrm{~s}, 2 \mathrm{H}), 2.91(\mathrm{~s}, 3 \mathrm{H}), 1.50(\mathrm{~s}, 9 \mathrm{H}) \mathrm{ppm}$.

$\underline{{ }^{13} \mathbf{C ~ N M R}}\left(101 \mathrm{MHz}, \mathrm{METHANOL}-d_{4}\right) \delta 159.3,158.1,137.3,133.8,132.4,131.0,130.5,128.6,128.2$, 126.1, 115.7, 114.1, 81.6, 54.2, 34.7, 28.9 ppm.

LC-MS (ESI) Calcd. for $\mathrm{C}_{20} \mathrm{H}_{24} \mathrm{~N}_{3} \mathrm{O}_{2}(\mathrm{M}+\mathrm{H})$ : 338.4, Found: 338.4 


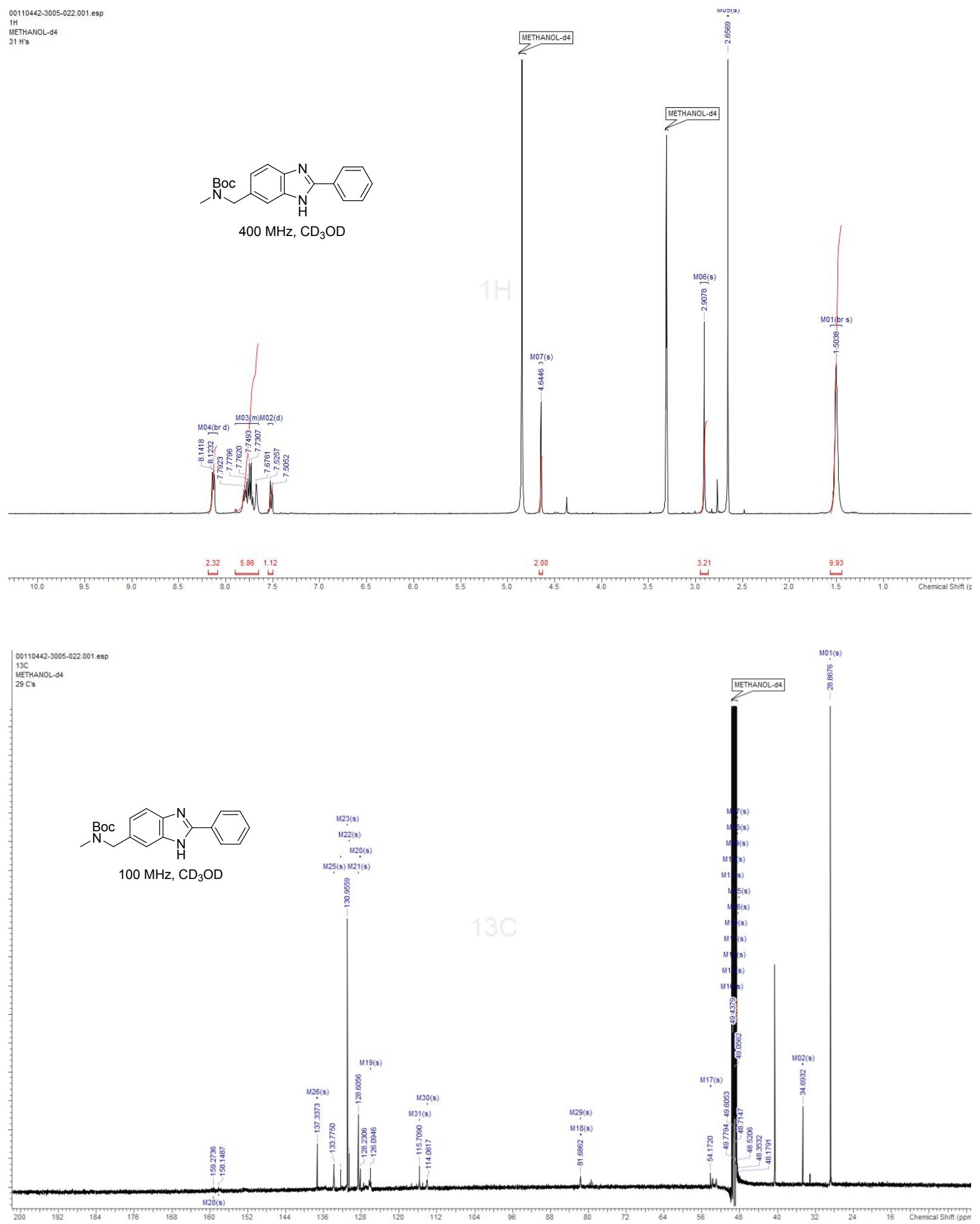




\section{2-phenyl-6-(pyridin-2-yl)-1H-benzo[d]imidazole (2m)}

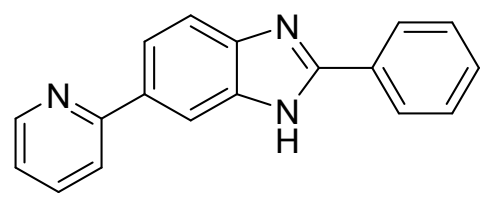

$\underline{1 \text { H NMR }}\left(400 \mathrm{MHz}\right.$, METHANOL- $\left.d_{4}\right) \delta \mathrm{ppm} 8.76(\mathrm{~d}, J=4.7 \mathrm{~Hz}, 1 \mathrm{H}) 8.40(\mathrm{~d}, J=1.2 \mathrm{~Hz}, 1 \mathrm{H}) 8.12-8.22$ $(\mathrm{m}, 5 \mathrm{H}) 7.93$ (d, $J=8.6 \mathrm{~Hz}, 1 \mathrm{H}) 7.71-7.80(\mathrm{~m}, 3 \mathrm{H}) 7.60$ (br t, $J=1.8 \mathrm{~Hz}, 1 \mathrm{H}) \mathrm{ppm}$.

${ }^{13} \mathbf{C}$ NMR $\left(101 \mathrm{MHz}\right.$, METHANOL- $\left.d_{4}\right) \delta$ ppm 155.53, 151.96, 147.48, 139.83, 135.00, 134.85, 132.82, $129.56,127.40,124.72,124.64,123.32,122.47,114.31,112.94,111.38$ ppm.

$\underline{\text { LC-MS }}$ (ESI) Calcd. for $\mathrm{C}_{18} \mathrm{H}_{13} \mathrm{~N}_{3}(\mathrm{M}+\mathrm{H})$ : 272.1, Found: 272.1 

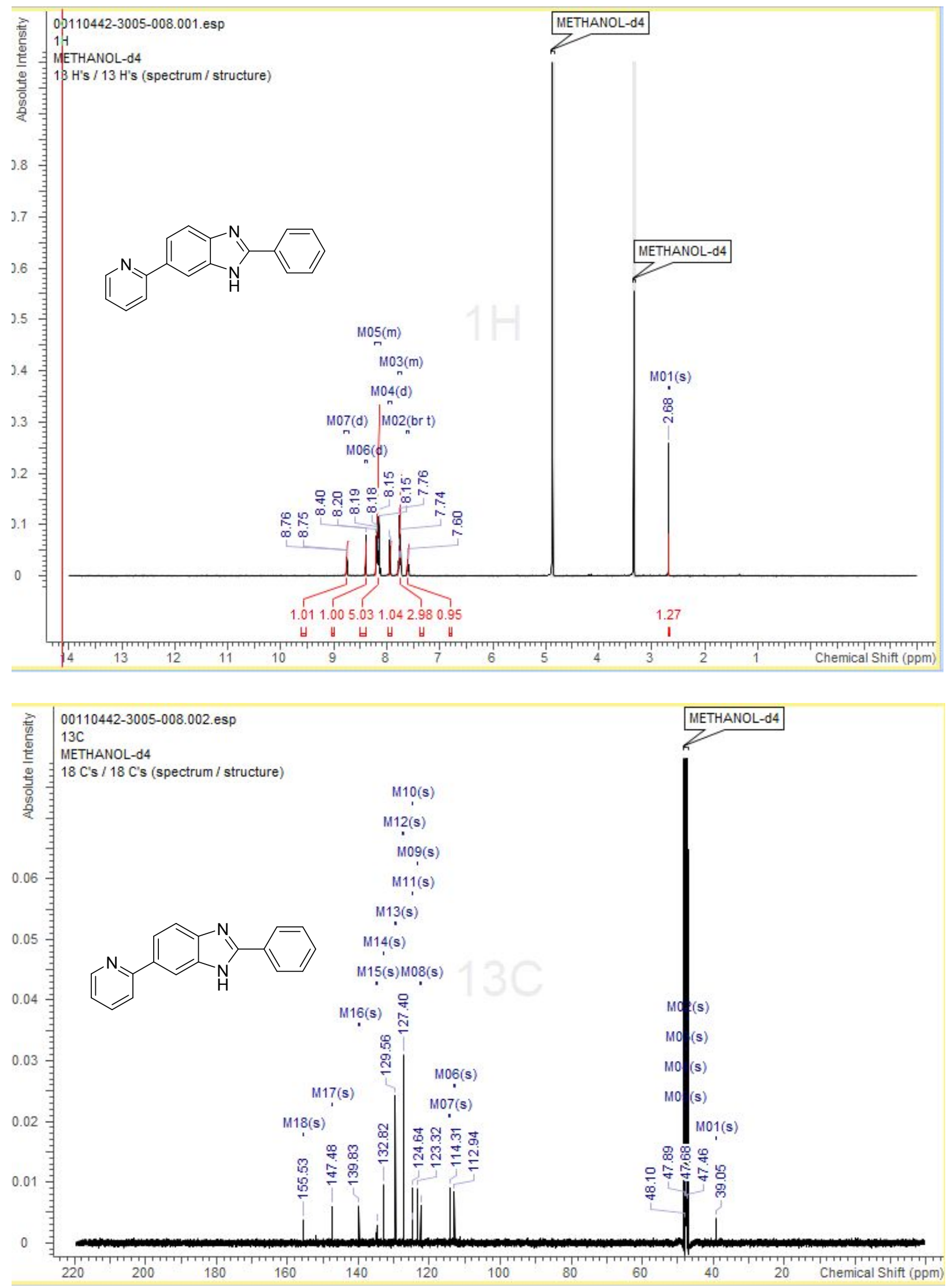


\section{1-benzyl-6-phenyl-1,7-dihydroimidazo[4,5-f]indazole (2n)}

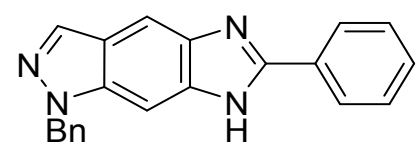

$\underline{\mathbf{1}_{\text {H NMR }}}\left(400 \mathrm{MHz}, \mathrm{METHANOL}-d_{4}\right) \delta 8.38(\mathrm{~s}, 1 \mathrm{H}), 8.11(\mathrm{~m}, 2 \mathrm{H}), 7.65$ - $7.75(\mathrm{~m}, 5 \mathrm{H}), 7.21$ - $7.31(\mathrm{~m}$,

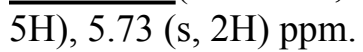

${ }^{13}$ C NMR $\left(101 \mathrm{MHz}\right.$, METHANOL- $\left.d_{4}\right) \delta 149.2,140.5,138.3,134.2,131.4,131.3,130.1,129.3,129.2$, 128.7, 128.6, 127.1, 125.8, 114.5, 112.5, 110.9, 54.5 ppm.

$\underline{\text { LC-MS }}$ (ESI) Calcd. for $\mathrm{C}_{21} \mathrm{H}_{17} \mathrm{~N}_{4}(\mathrm{M}+\mathrm{H}): 325.4$, Found: 325.4 
<smiles></smiles>
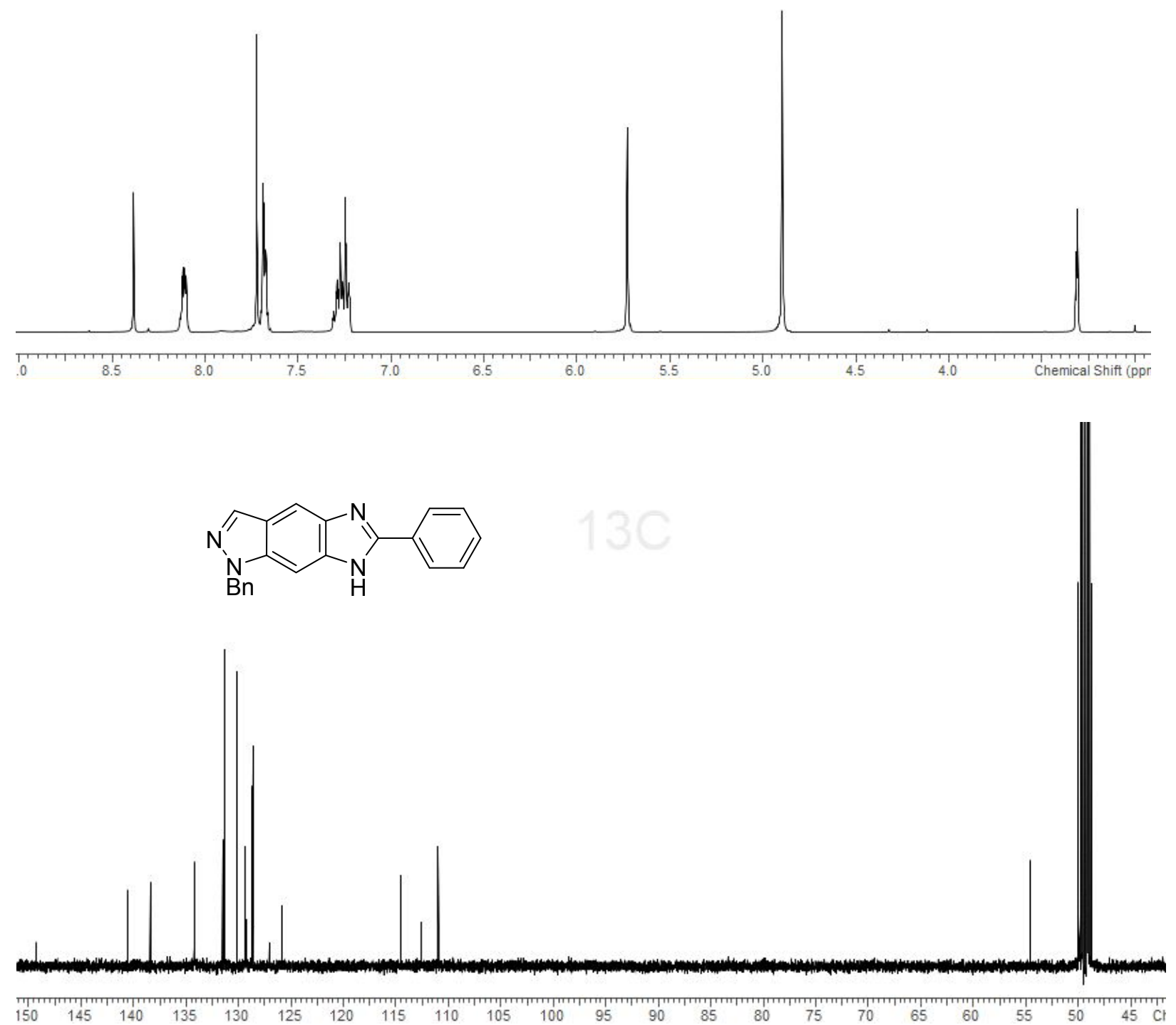


\section{7-phenyl-6H-imidazo[4',5':3,4] benzo[1,2-d]thiazole (2o)}

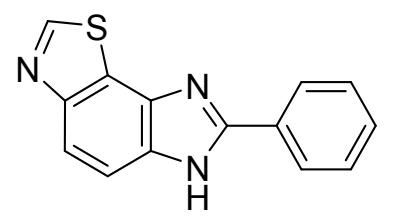

1H NMR $\left(400 \mathrm{MHz}\right.$, METHANOL- $\left.d_{4}\right) \delta 9.29(\mathrm{~s}, 1 \mathrm{H}), 8.10-8.20(\mathrm{~m}, 3 \mathrm{H}), 7.84(\mathrm{~d}, J=8.98 \mathrm{~Hz}, 1 \mathrm{H})$, $7.62-7.70$ (m, $3 \mathrm{H}) \mathrm{ppm}$.

${ }^{{ }^{13} \mathbf{C ~ N M R}}{ }^{13} \mathrm{C}$ NMR $\left(101 \mathrm{MHz}\right.$, METHANOL- $\left.d_{4}\right) \delta 155.5,153.1,152.8,133.5,133.2,133.0,130.8$, $128.5,128.0,122.1,121.1,113.4 \mathrm{ppm}$.

$\underline{\text { LC-MS }}$ (ESI) Calcd. for $\mathrm{C}_{14} \mathrm{H}_{10} \mathrm{~N}_{3} \mathrm{~S}(\mathrm{M}+\mathrm{H}): 252.3$, Found: 252.3 


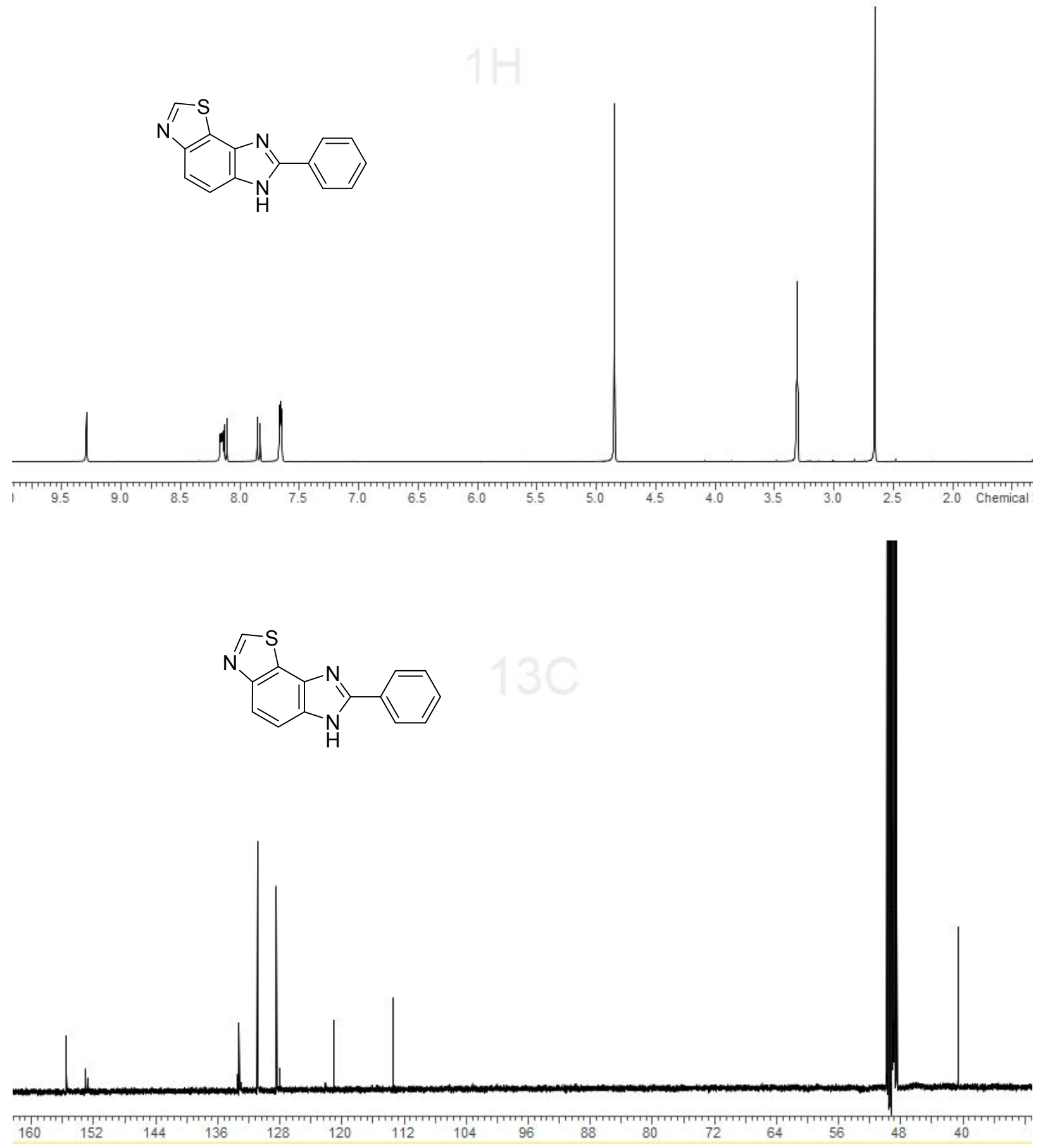




\section{1-(2-phenyl-6,7-dihydroimidazo[4,5-g]indol-8(1H)-yl)ethan-1-one (2p)}

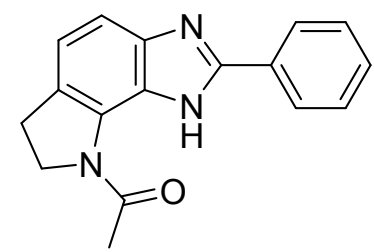

$\underline{{ }^{1} \text { H NMR }}\left(400 \mathrm{MHz}\right.$, METHANOL- $\left.d_{4}\right) \delta 8.12(\mathrm{~s}, 1 \mathrm{H}) 8.10(\mathrm{~d}, J=1.56 \mathrm{~Hz}, 1 \mathrm{H}) 7.71-7.82(\mathrm{~m}, 3 \mathrm{H}) 7.49$ $7.52(\mathrm{~m}, 1 \mathrm{H}) 7.47(\mathrm{~s}, 1 \mathrm{H}) 4.36(\mathrm{t}, J=8.78 \mathrm{~Hz}, 2 \mathrm{H}) 3.45(\mathrm{t}, J=8.59 \mathrm{~Hz}, \mathrm{H}) 2.40(\mathrm{~s}, 3 \mathrm{H}) \mathrm{ppm}$.

$\underline{{ }^{13} \mathbf{C ~ N M R}}\left(101 \mathrm{MHz}\right.$, METHANOL- $\left.d_{4}\right) \delta 170.2,147.7,134.1,133.3,129.8,129.7,128.0,127.4,123.0$, $120.6,109.8,100.0,49.8,28.3,22.0 \mathrm{ppm}$.

$\underline{\text { LC-MS }}$ (ESI) Calcd. for $\mathrm{C}_{17} \mathrm{H}_{15} \mathrm{~N}_{3} \mathrm{O}(\mathrm{M}+\mathrm{H})$ : 278.1, Found: 278.5

$\underline{\text { Regioselectivity }}>20: 1$ - Assigned by COSY 


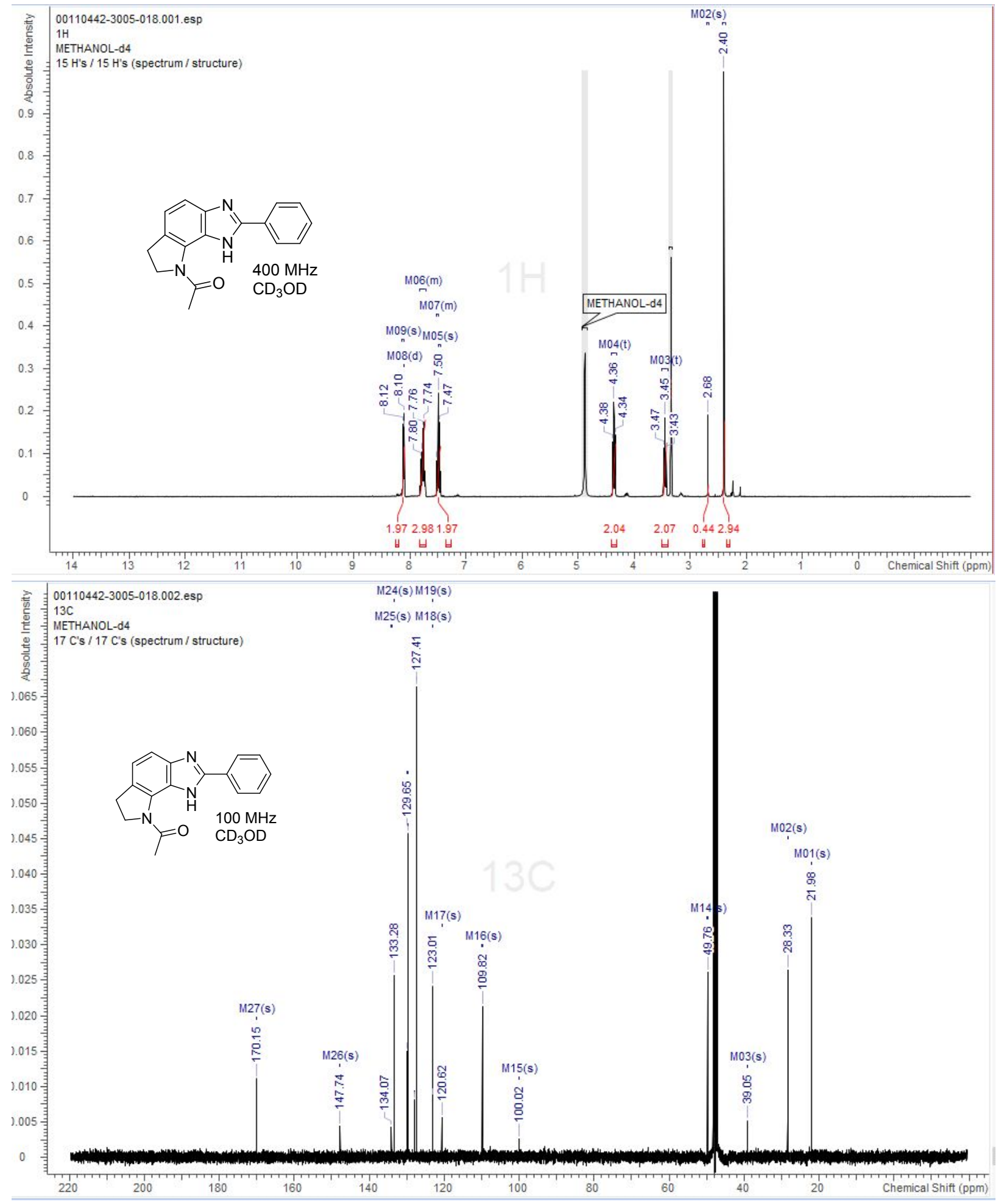


cosy DMSO \{C: \Bruker \TopSpin3.0\} schmid24 34

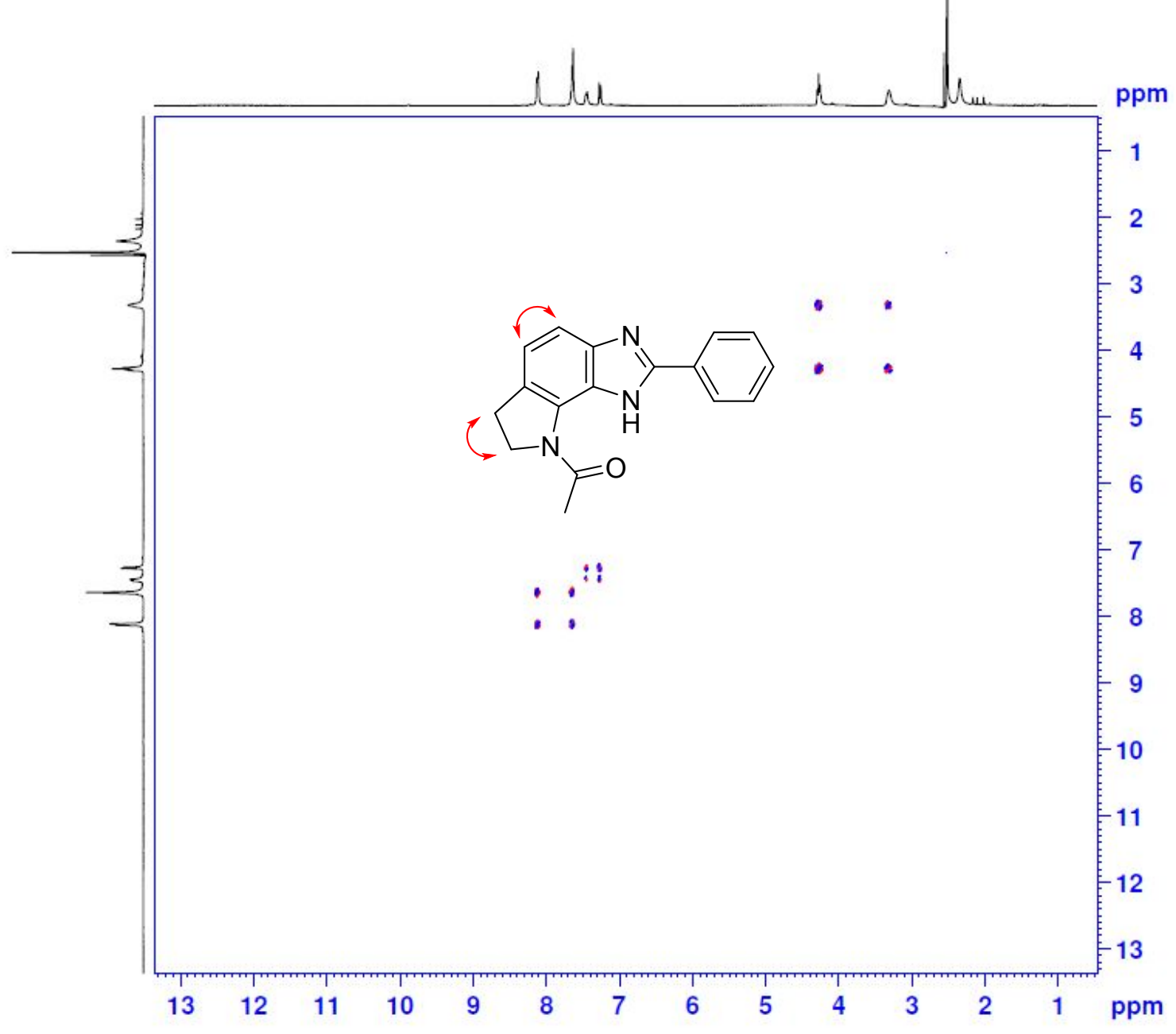




\section{Spectroscopic Data for Scheme 3}

Compounds were prepared in parallel format via General Library Procedure B.

\section{5-fluoro-1-methyl-2-phenyl-1H-benzo[d]imidazole (4a)}

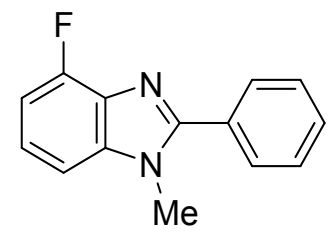

$\underline{{ }^{1} \text { H NMR }}\left(400 \mathrm{MHz}\right.$, METHANOL- $\left.d_{4}\right) \delta 7.81$ - $7.95(\mathrm{~m}, 2 \mathrm{H}) 7.59$ - $7.76(\mathrm{~m}, 4 \mathrm{H}) 7.51$ (td, $J=8.20,4.68$ $\mathrm{Hz}, 1 \mathrm{H}) 7.28$ (t, $J=9.26 \mathrm{~Hz}, 1 \mathrm{H}) 4.01(\mathrm{~s}, 3 \mathrm{H}) \mathrm{ppm}$.

13C NMR (101 MHz, METHANOL- $d_{4}$ ) (C-F coupling observed) $\delta 154.7,154.3,151.8,139.4,139.3$, 133.6, 131.4, 130.8, 127.2, 127.1, 127.1, 111.8, 111.6, 109.5, 109.4, 33.5 ppm.

$\underline{\text { LC-MS }}$ (ESI) Calcd. for $\mathrm{C}_{14} \mathrm{H}_{12} \mathrm{FN}_{2}(\mathrm{M}+\mathrm{H})$ : 227.3, Found: 227.3 


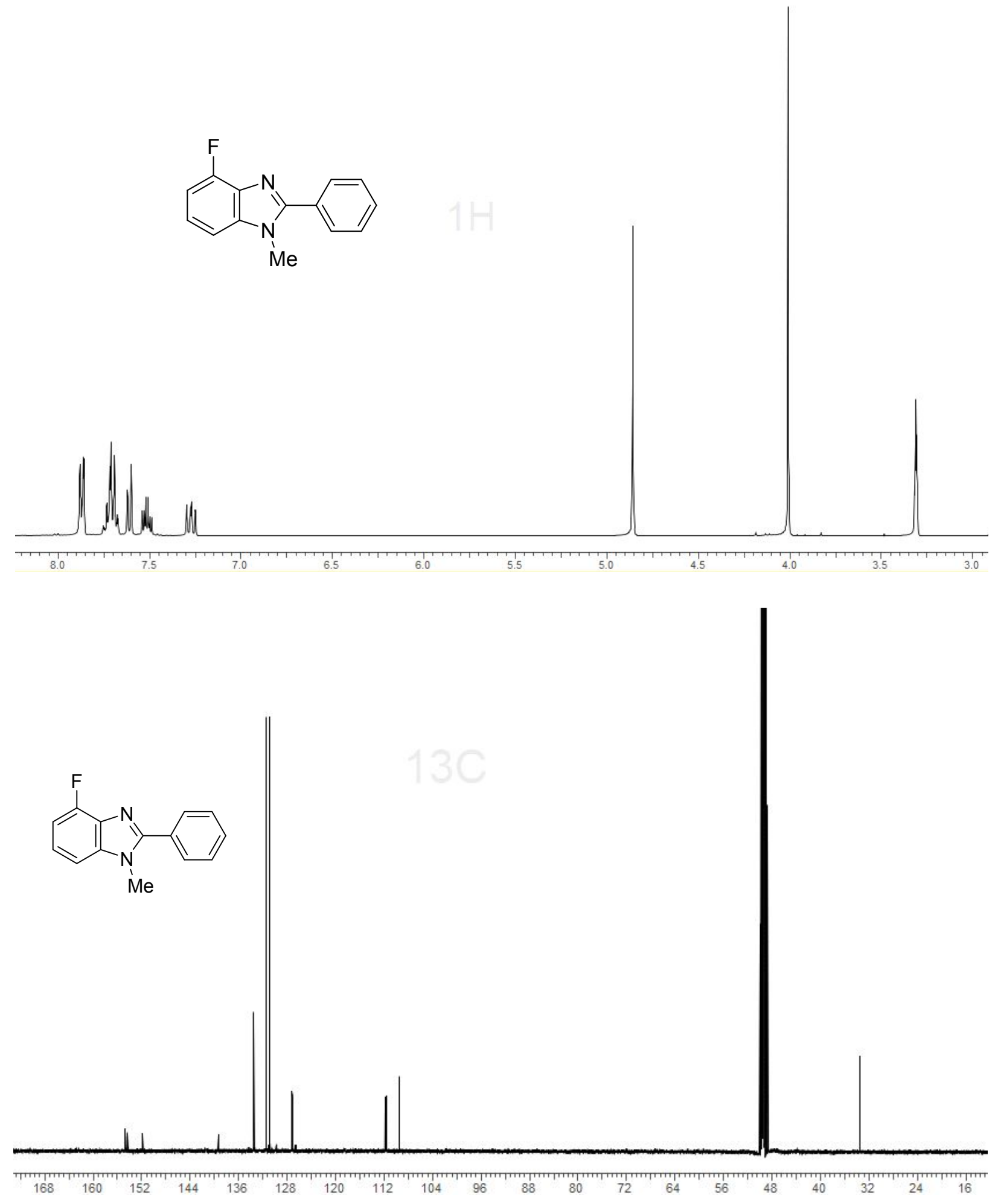




\section{4-methoxy-1-methyl-2-phenyl-1H-benzo[d]imidazole (4b)}

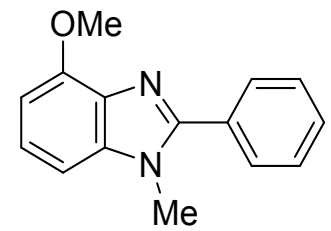

1H NMR $\left(500 \mathrm{MHz}\right.$, METHANOL- $\left.d_{4}\right) \delta 7.86-7.95(\mathrm{~m}, 2 \mathrm{H}), 7.70-7.85(\mathrm{~m}, 3 \mathrm{H}), 7.63(\mathrm{t}, J=8.19 \mathrm{~Hz}, 1$ H), $7.49(\mathrm{~d}, J=8.07 \mathrm{~Hz}, 1 \mathrm{H}), 7.21$ (d, $J=8.07 \mathrm{~Hz}, 1 \mathrm{H}), 4.10$ (s, $3 \mathrm{H}), 4.06$ (s, $3 \mathrm{H}) \mathrm{ppm}$.

$\frac{{ }^{13} \text { C NMR }}{1086}$ MHz, METHANOL- $\left.d_{4}\right) \delta 151.6,149.4,136.5,134.6,131.5,131.1,129.2,124.1,123.1$, 108.6, 106.1, 57.2, $33.6 \mathrm{ppm}$.

$\underline{\text { LC-MS }}$ (ESI) Calcd. for $\mathrm{C}_{15} \mathrm{H}_{15} \mathrm{~N}_{2 \mathrm{O}}(\mathrm{M}+\mathrm{H}): 239.3$, Found: 239.4 

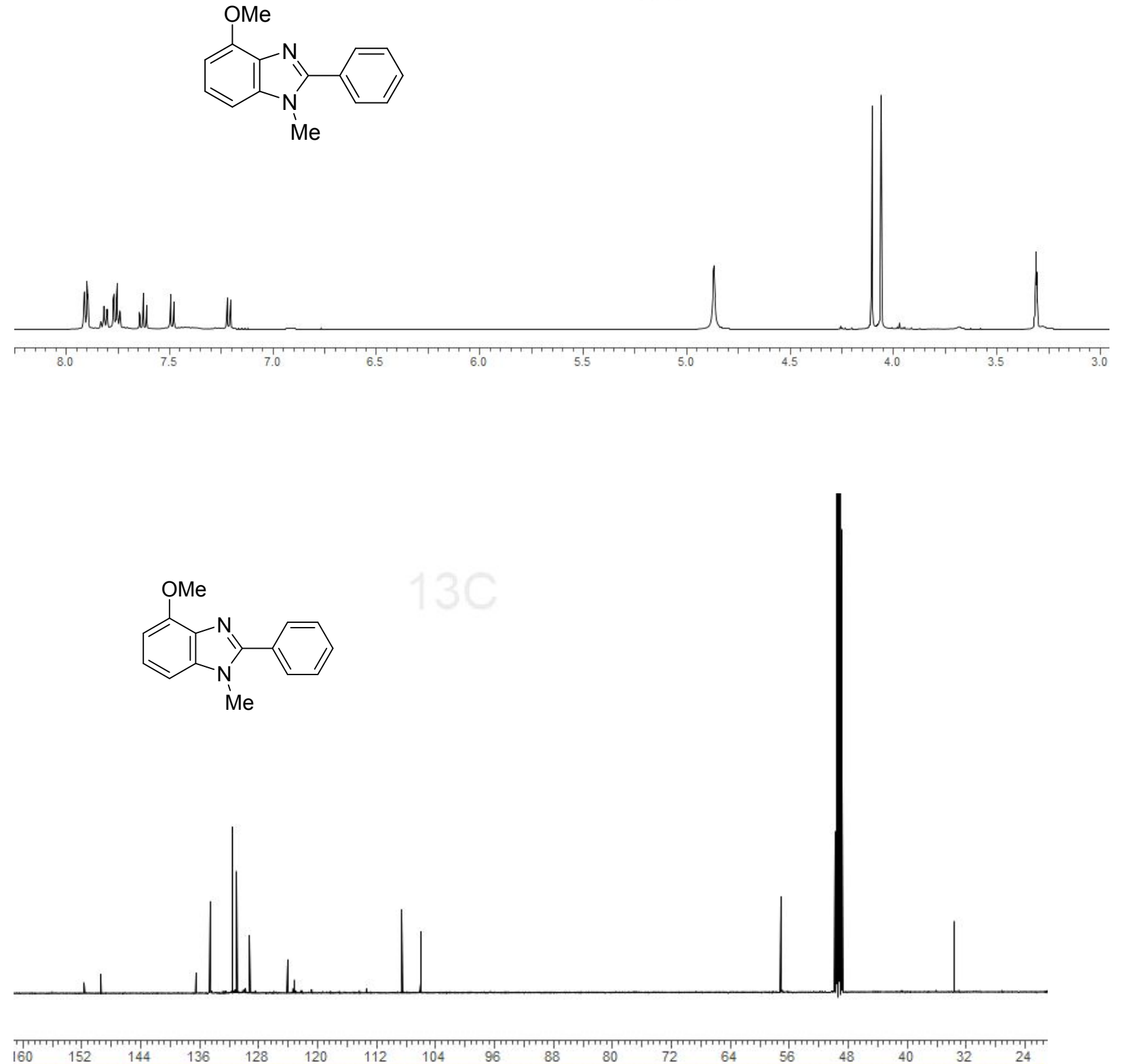


\section{1,5,7-trimethyl-2-phenyl-1H-benzo[d]imidazole (4c)}

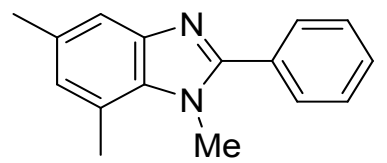

$\underline{\text { 1H NMR }}\left(400 \mathrm{MHz}, \mathrm{METHANOL}-d_{4}\right) \delta 7.72-7.91(\mathrm{~m}, 5 \mathrm{H}), 7.45(\mathrm{~s}, 1 \mathrm{H}), 7.31(\mathrm{~s}, 1 \mathrm{H}), 4.22(\mathrm{~s}, 3 \mathrm{H})$, $2.91(\mathrm{~s}, 3 \mathrm{H}), 2.57$ (s, $3 \mathrm{H}) \mathrm{ppm}$.

$\underline{{ }^{13} \mathbf{C ~ N M R}}\left(101 \mathrm{MHz}, \mathrm{METHANOL}-d_{4}\right) \delta 151.7,139.3,134.4,133.0,131.8,131.4,131.9,131.0,126.4$, $124.1,112.5,36.2,21.5,18.8 \mathrm{ppm}$.

$\underline{\text { LC-MS }}$ (ESI) Calcd. for $\mathrm{C}_{16} \mathrm{H}_{17} \mathrm{~N}_{2}(\mathrm{M}+\mathrm{H})$ : 237.1, Found: 237.4 

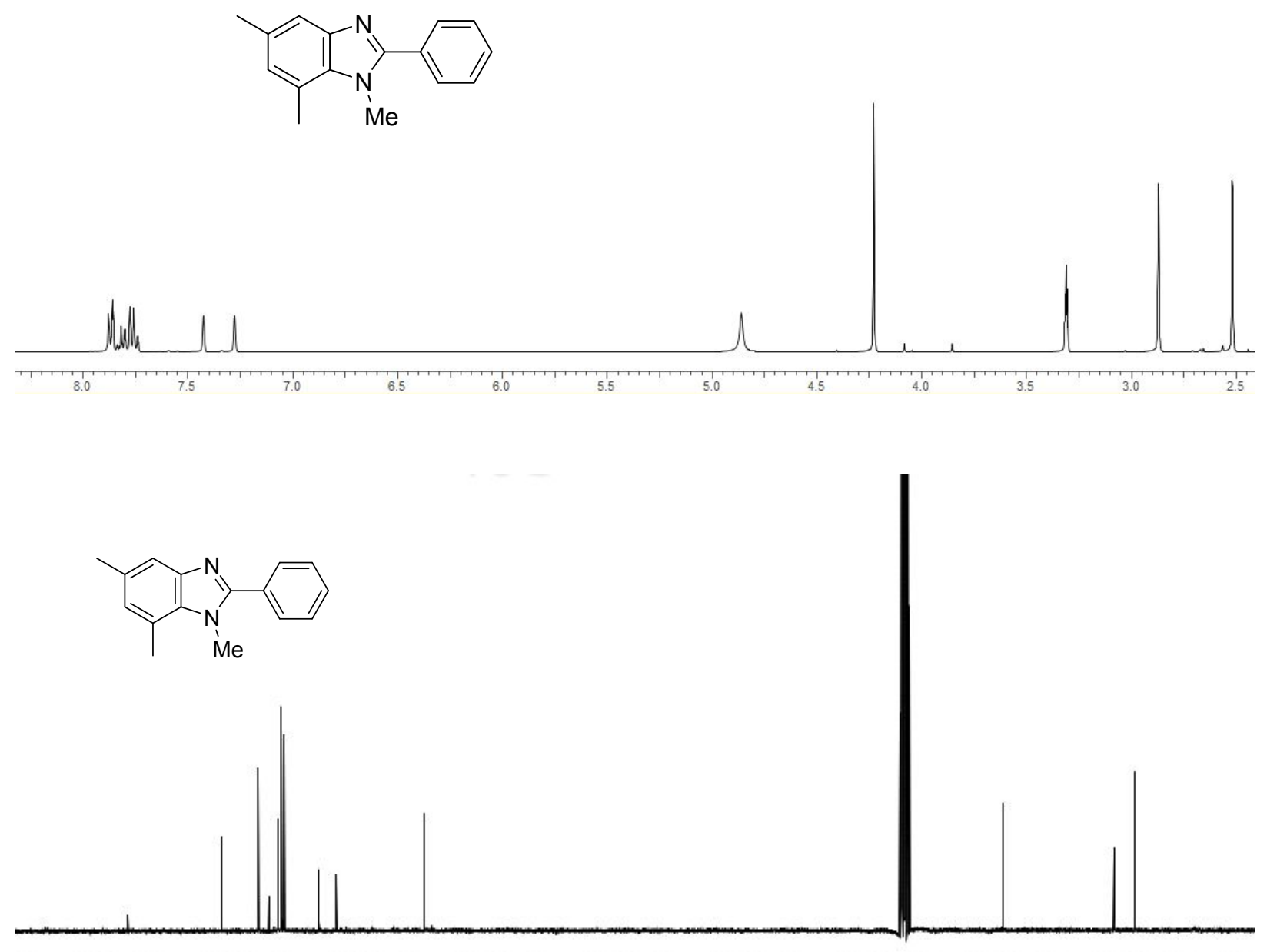

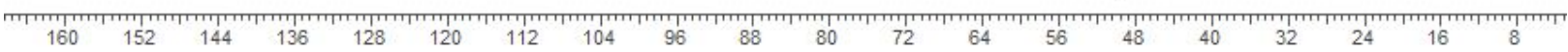


7-fluoro-1-methyl-2-phenyl-1H-benzo[d]imidazole (4d)

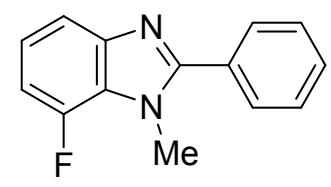

1H NMR $\left(500 \mathrm{MHz}\right.$, METHANOL- $\left.d_{4}\right) \delta 7.89(\mathrm{~d}, J=7.18 \mathrm{~Hz}, 2 \mathrm{H}) 7.71-7.81(\mathrm{~m}, 3 \mathrm{H}) 7.59$ - $7.65(\mathrm{~m}, 1$ H) $7.51-7.59$ (m, 1 H) 7.37 (ddd, $J=11.74,8.07,0.73 \mathrm{~Hz}, 1 \mathrm{H}) 4.18$ (d, $J=1.47 \mathrm{~Hz}, 3 \mathrm{H}) \mathrm{ppm}$.

${ }^{13} \mathbf{C ~ N M R}\left(126 \mathrm{MHz}, \mathrm{METHANOL}-d_{4}\right)$ (C-F coupling observed) $\delta 154.0,152.4,150.4,137.8$, $134.1,131.3,130.8,128.0,127.9,125.1,124.2,124.1,112.9,112.8,35.6$ (2 peaks) ppm.

LC-MS (ESI) Calcd. for $\mathrm{C}_{14} \mathrm{H}_{11} \mathrm{FN}_{2}(\mathrm{M}+\mathrm{H})$ : 227.3, Found: 227.3

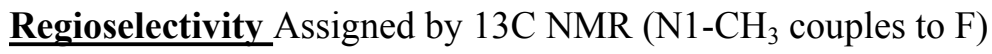



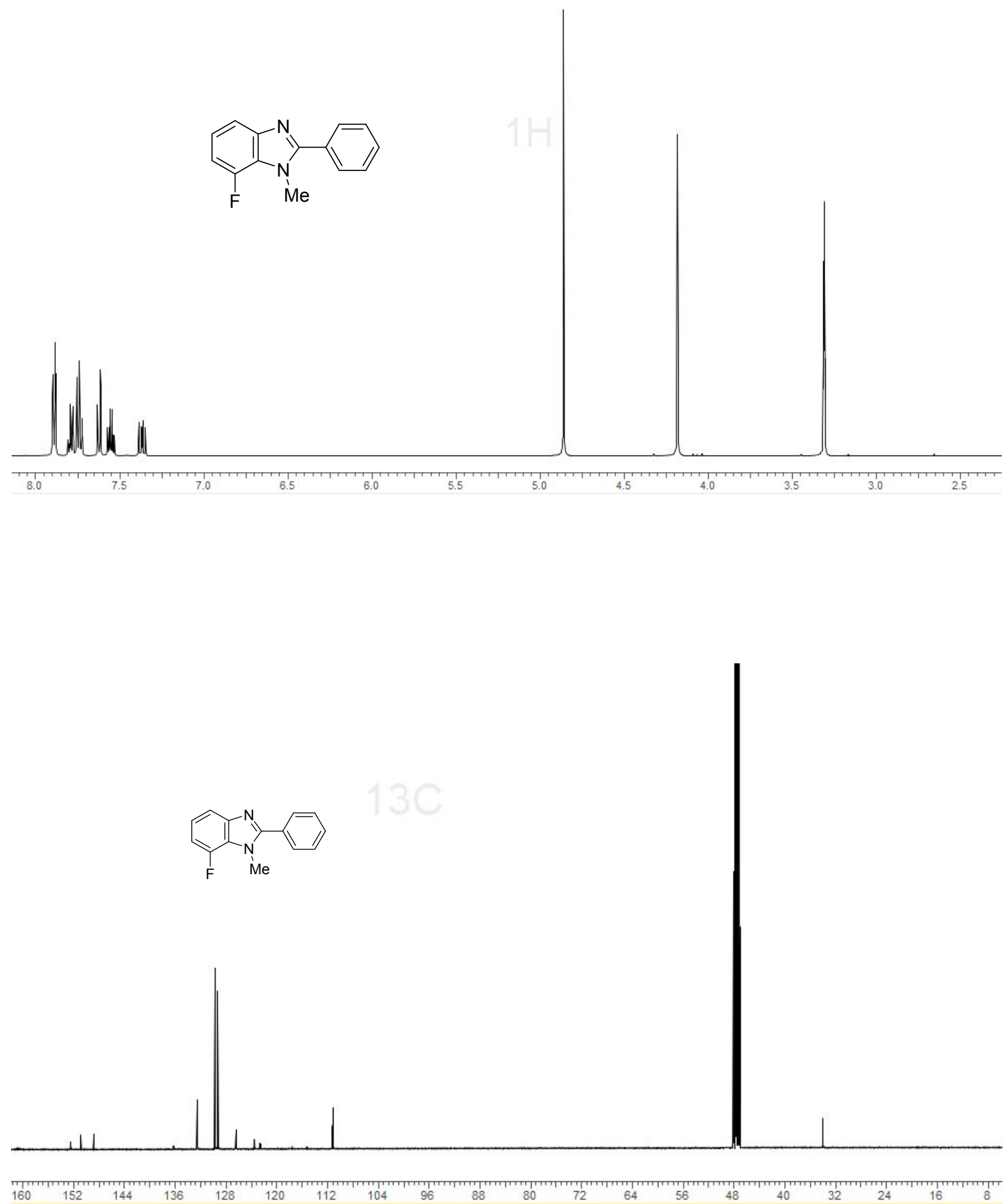


\section{6-methoxy-1-methyl-2-phenyl-1H-benzo[d]imidazole (4e)}

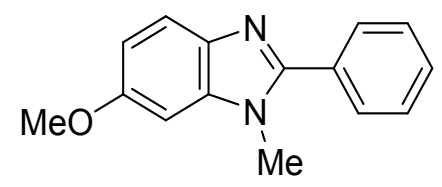

1H NMR $\left(500 \mathrm{MHz}\right.$, METHANOL- $\left.d_{4}\right) \delta 7.93(\mathrm{~d}, J=6.83 \mathrm{~Hz}, 2 \mathrm{H}), 7.75-7.98(\mathrm{~m}, 4 \mathrm{H}), 7.41(\mathrm{~d}, J=2.45$ $\mathrm{Hz}, 1 \mathrm{H}), 7.36$ (m, $1 \mathrm{H}), 4.1$ (s, $3 \mathrm{H}), 3.98$ (s, $3 \mathrm{H}) \mathrm{ppm}$.

$\frac{{ }^{13} \mathbf{C ~ N M R}}{\left(126 \mathrm{MHz}, \mathrm{METHANOL}-d_{4}\right)} \delta 161.0$ 151.2, 136.2, 134.4, 131.1, 131.0, 124.2, 118.6, 116.0, 96.5, 56.9, $33.3 \mathrm{ppm}$.

$\underline{\text { LC-MS }}$ (ESI) Calcd. for $\mathrm{C}_{15} \mathrm{H}_{15} \mathrm{~N}_{2 \mathrm{O}}(\mathrm{M}+\mathrm{H}): 239.3$, Found: 239.3 


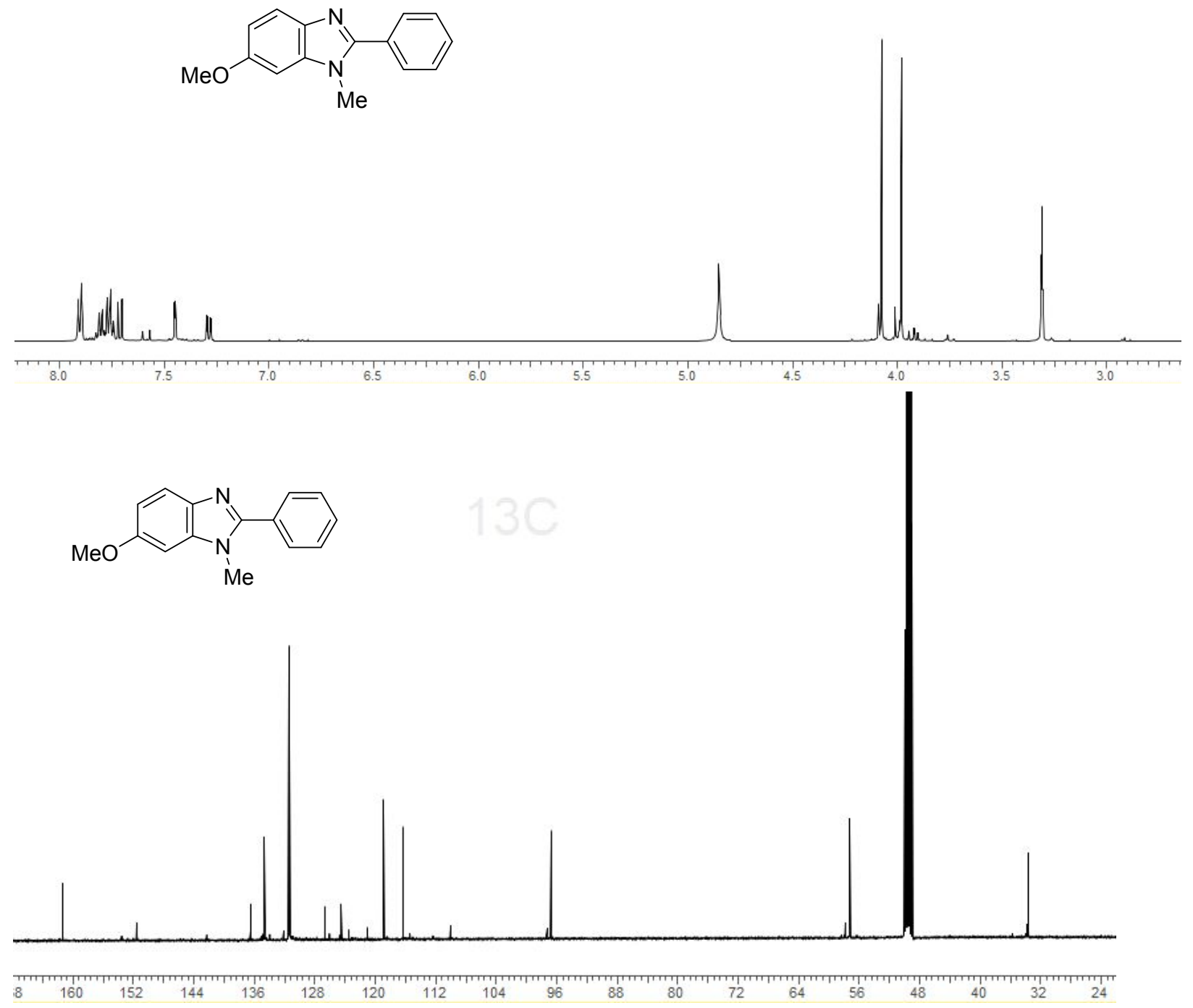




\section{Preparative scale sequences (Scheme 4) - experimental procedures}

Preparative scale synthesis of compound 2e

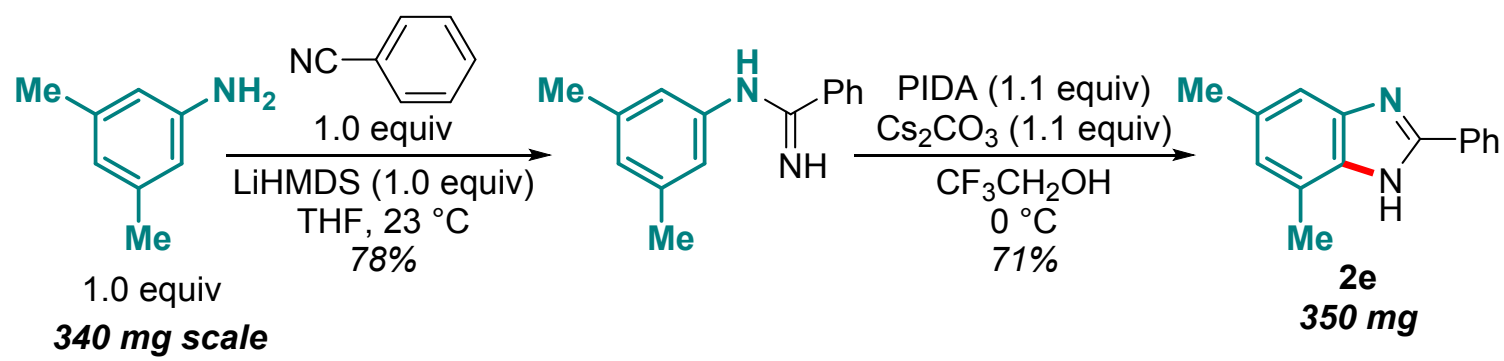

Amidine formation - To 3,5-dimethylaniline (340 mg, $2.81 \mathrm{mmol}, 1.0$ equiv) in a 3-neck round bottom flask fitted with an addition funnel was added THF $(17 \mathrm{~mL})$. The flask was cooled to $0{ }^{\circ} \mathrm{C}$ in an ice bath and LiHMDS ( $1 \mathrm{M}$ in THF, $2.81 \mathrm{~mL}, 2.81 \mathrm{mmol}, 1.0$ equiv) was added dropwise via addition funnel. The reaction was stirred for $20 \mathrm{~min}$ at $0{ }^{\circ} \mathrm{C}$, then benzonitrile $(286 \mathrm{mg}, 2.81 \mathrm{mmol}, 1.0$ equiv) was added. The reaction was warmed to $23{ }^{\circ} \mathrm{C}$ and stirred for $17 \mathrm{~h}$. Water $(30 \mathrm{~mL})$ was added and the mixture was extracted $2 \mathrm{X}$ with EtOAc. The combined organic phases were dried over $\mathrm{Na}_{2} \mathrm{SO}_{4}$, filtered, and concentrated to provide crude amidine intermediate $(492 \mathrm{mg}, 2.20 \mathrm{mmol}, 78 \%$ yield) that was used in the subsequent step without purification.

Oxidative cyclization - To the 2-dram vial containing amidine intermediate $(2.20 \mathrm{mmol})$ under air atmosphere was added $\mathrm{Cs}_{2} \mathrm{CO}_{3}(792 \mathrm{mg}, 2.43 \mathrm{mmol}, 1.1$ equiv) and 2,2,2-trifluoroethanol $(2.0 \mathrm{~mL})$. Cooled to $0{ }^{\circ} \mathrm{C}$ and added PIDA (783 mg, $2.43 \mathrm{mmol}, 1.1$ equiv). After stirring for $1.5 \mathrm{~h}$, solvent was removed in vacuo. The crude mixture was diluted with EtOAc and water; the organic phase was separated, dried over $\mathrm{Na}_{2} \mathrm{SO}_{4}$, filtered, and concentrated in vacuo. The residue was chromatographed $(20 \% \mathrm{EtOAc} / \mathrm{heptanes}$ to $50 \%$ EtOAc/heptanes) to provide $2 \mathrm{e}$ (350 $\mathrm{mg}, 1.58 \mathrm{mmol}, 71 \%$ yield).

Preparative scale synthesis of compound 4e:

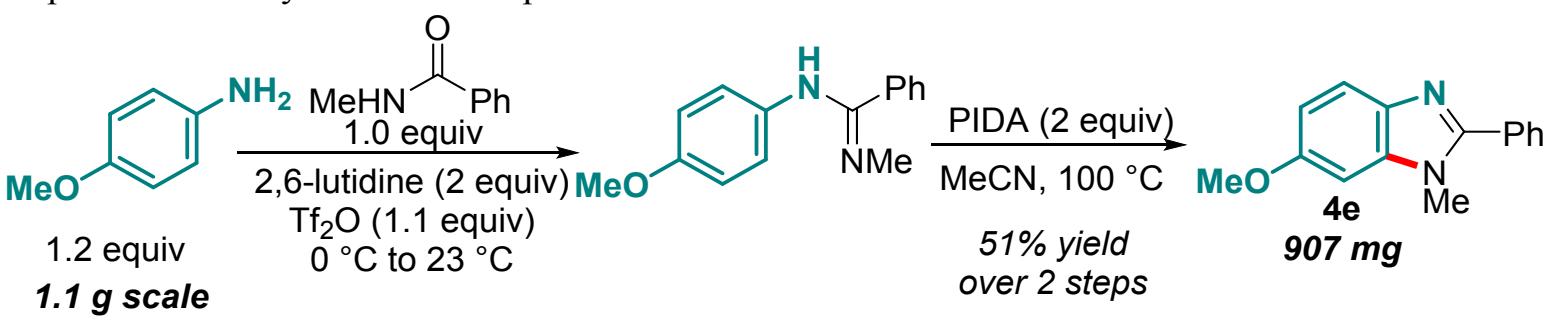

Amidine formation - N-methylbenzamide (1.01 g, $7.5 \mathrm{mmol}, 1.0$ equiv) was dissolved in $20 \mathrm{~mL}$ dry dichloromethane in a $125 \mathrm{~mL}$ round-bottom flask. 2,6-lutidine $(1.9 \mathrm{~mL}, 16.5 \mathrm{mmol}, 2.2$ equiv) was then added drop-wise at $23{ }^{\circ} \mathrm{C}$, under nitrogen. Reaction flask was then cooled in an ice-water bath, and trifluoromethanesulfonic anhydride $(1.76 \mathrm{~mL}, 8.3 \mathrm{mmol}, 1.1$ equiv) was added drop-wise. The darkpink/wine color solution generated was stirred under nitrogen, and gradually warmed to $23{ }^{\circ} \mathrm{C}$ over $2.5 \mathrm{~h}$. A solution of 4-methoxyaniline $(1.1 \mathrm{~g}, 9.0 \mathrm{mmol}, 1.2 \mathrm{eq}$.) in $5 \mathrm{~mL}$ dry dichloromethane was then added to the reaction flask, and contents were stirred overnight at $23{ }^{\circ} \mathrm{C}$. Reaction was quenched by adding $10 \mathrm{~mL}$ of water. Organic layer was separated, and aqueous layer was extracted with $2 \times 15 \mathrm{~mL}$ of ethyl acetate. Combined organic layers were then dried over sodium sulfate, concentrated and purified using silica gel ISCO Combiflash chromatography (0 to $100 \%$ ethyl acetate in heptane). Partially pure amidine (1.8 g, $100 \%$ ) was obtained, which was used as is in the subsequent step.

Oxidative cyclization - amidine intermediate was dissolved in dry acetonitrile $(38 \mathrm{~mL}, \mathrm{c}=0.20 \mathrm{M})$, and transferred to a sealable tube. Diacetoxyiodobenzene (4.80 g, $14.9 \mathrm{mmol}, 2.0$ equiv) was added in one 
portion. The reaction vessel was sealed and heated at $100{ }^{\circ} \mathrm{C}$ for $2 \mathrm{~h}$. After cooling to $23{ }^{\circ} \mathrm{C}$, the mixture was diluted with $40 \mathrm{~mL}$ EtOAc and washed with $40 \mathrm{~mL}$ water. The aqueous layer was extracted with $2 \mathrm{x}$ $30 \mathrm{~mL}$ ethyl acetate. Combined organics were dried, concentrated and purified using ISCO (0 to $100 \%$ ethyl acetate in heptane) to give 6-methoxy-1-methyl-2-phenyl-1H-benzo[d]imidazole (907 mg, 51\% yield over 2 steps). 


\section{Spectroscopic Data for Scheme 5}

Compounds were prepared in parallel format via General Library Procedure A.

Compounds $\mathbf{6 a}, \mathbf{6 b}$, and $\mathbf{6 e}$ have been fully characterized previously and NMR spectra were matched to reported values:

6a) Dubey, P. K.; Chowdary, K. S.; Ramesh, B.; Prasada Reddy, P. V. V. Synth. Commun. 2010, 40, 697708.

6b) Mishra, A.; Sahu, S.; Dash, N.; Behera, S. K.; Krishnamoorthy, G. J. Phys Chem. B. 2013, 117, 94699477.

6e) Kulkami, S. S.; Newman, A. H. Bioorganic \& Medicinal Chemistry Letters 2007, 17, 2987-2991.

\section{6-(cyclopropylmethoxy)-2-phenyl-3H-imidazo[4,5-c]pyridine (6d)}

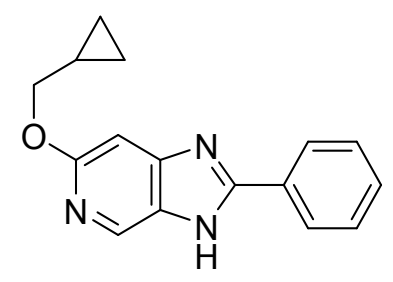

$\underline{{ }^{1} \mathbf{H} \text { NMR }}\left(400 \mathrm{MHz}, \mathrm{CD}_{3} \mathrm{OD}\right): \delta 8.58(\mathrm{~s}, 1 \mathrm{H}), 8.15-8.10(\mathrm{~m}, 2 \mathrm{H}), 7.62-7.57(\mathrm{~m}, 3 \mathrm{H}), 7.14(\mathrm{~s}, 1 \mathrm{H}), 4.19(\mathrm{~d}$, $J=7.0 \mathrm{~Hz}, 2 \mathrm{H}), 1.38-1.33(\mathrm{~m}, 1 \mathrm{H}), 0.70-0.66(\mathrm{~m}, 2 \mathrm{H}), 0.46-0.42(\mathrm{~m}, 2 \mathrm{H}) \mathrm{ppm}$.

$\frac{{ }^{13} \mathrm{C} \text { NMR }}{3.8 \mathrm{ppm}}\left(100 \mathrm{MHz}, \mathrm{CD}_{3} \mathrm{OD}\right): \delta 160.6,157.9,148.3,136.3,134.3,132.9,130.5,128.5,92.8,74.5,11.1$, $3.8 \mathrm{ppm}$.

$\underline{\text { LC-MS }}$ (ESI) Calcd. for $\mathrm{C}_{16} \mathrm{H}_{15} \mathrm{~N}_{3} \mathrm{O}(\mathrm{M}+\mathrm{H})$ : 266.1, Found: 266.3 .

Regioselectivity $2: 1$ - Assigned by ${ }^{1} \mathrm{HNMR}$. 
METHANOL-A4

METHANOL-d4
$14 \mathrm{HS} / 15 \mathrm{HS}$ s spectrum/ structure
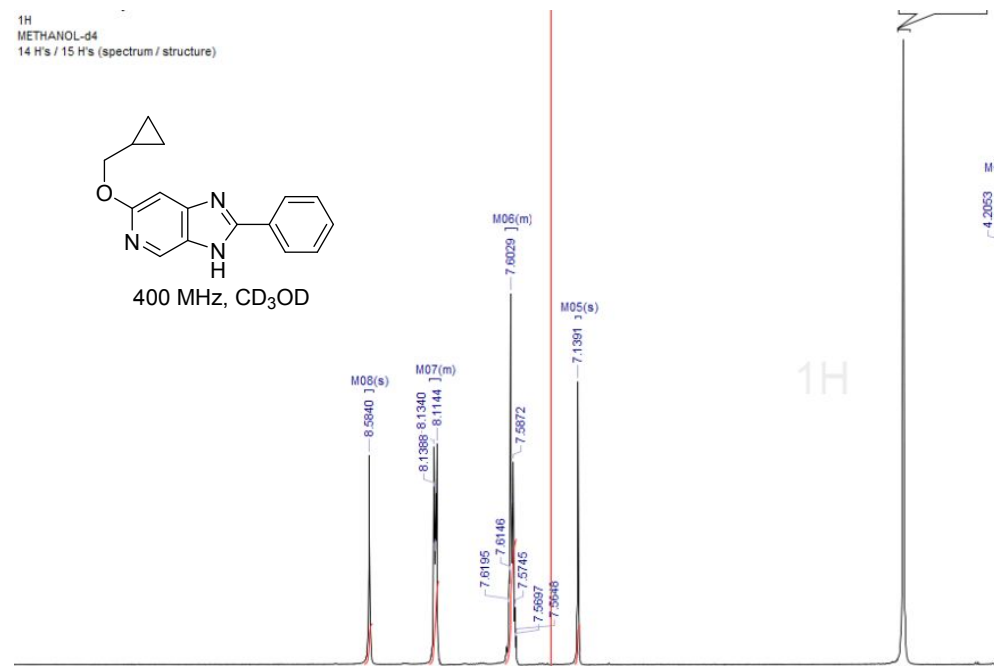

Mo4i

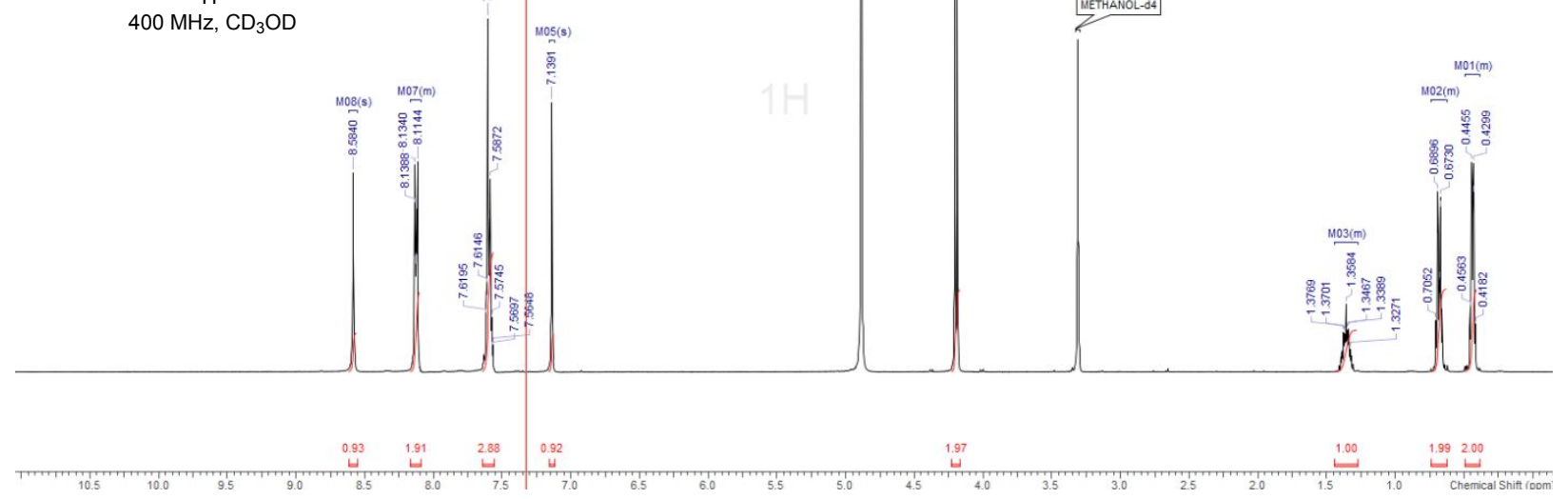

26 - major - -13 C. $\operatorname{lnk}$
$13 \mathrm{C}$
METHANOL-D4
22 CS

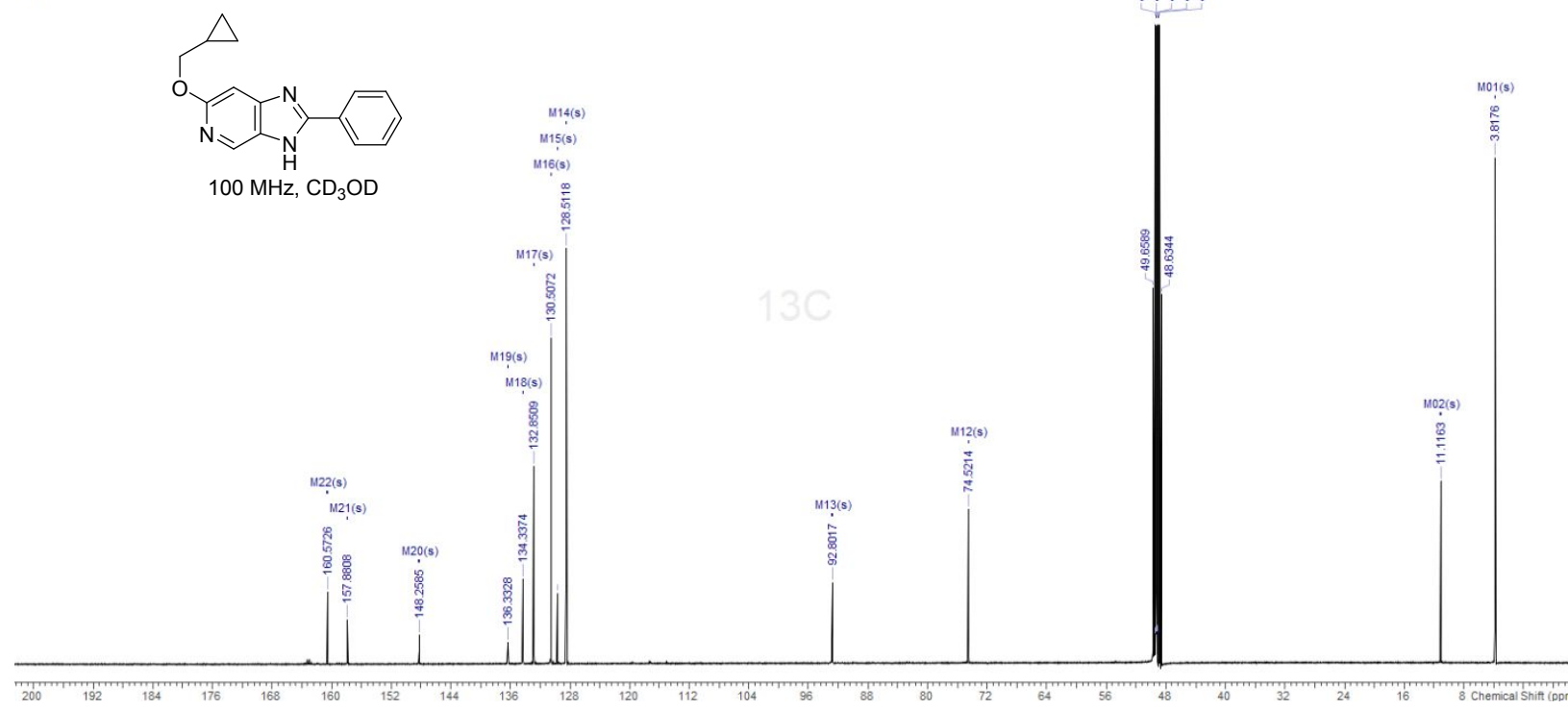




\section{6-methyl-2-phenyl-3H-imidazo[4,5-c]pyridine (6f)}

(from 2-methylpyridin-4-amine)

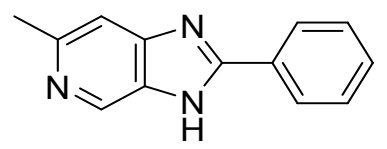

$\underline{{ }^{1} \mathbf{H} \text { NMR }}\left(400 \mathrm{MHz}, \mathrm{CD}_{3} \mathrm{OD}\right): \delta 8.73(\mathrm{~s}, 1 \mathrm{H}), 8.13-8.10(\mathrm{~m}, 2 \mathrm{H}), 7.56-7.55(\mathrm{~m}, 3 \mathrm{H}), 7.44(\mathrm{~s}, 1 \mathrm{H}), 2.63(\mathrm{~s}$, 3H) ppm.

${ }^{13} \mathbf{C}$ NMR $\left(100 \mathrm{MHz}, \mathrm{CD}_{3} \mathrm{OD}\right):($ tautomeric mix) $\delta 157.4,151.2,140.7,133.2,132.4$ (2 peaks), 130.6, $130.5,130.4,129.8,129.7,128.5,23.6 \mathrm{ppm}$.

LC-MS (ESI) Calcd. for $\mathrm{C}_{13} \mathrm{H}_{11} \mathrm{~N}_{3}(\mathrm{M}+\mathrm{H})$ : 210.1, Found: 210.1 .

$\underline{\text { Regioselectivity }}>20: 1$ - Assigned by ${ }^{1} \mathrm{HNMR}$. 

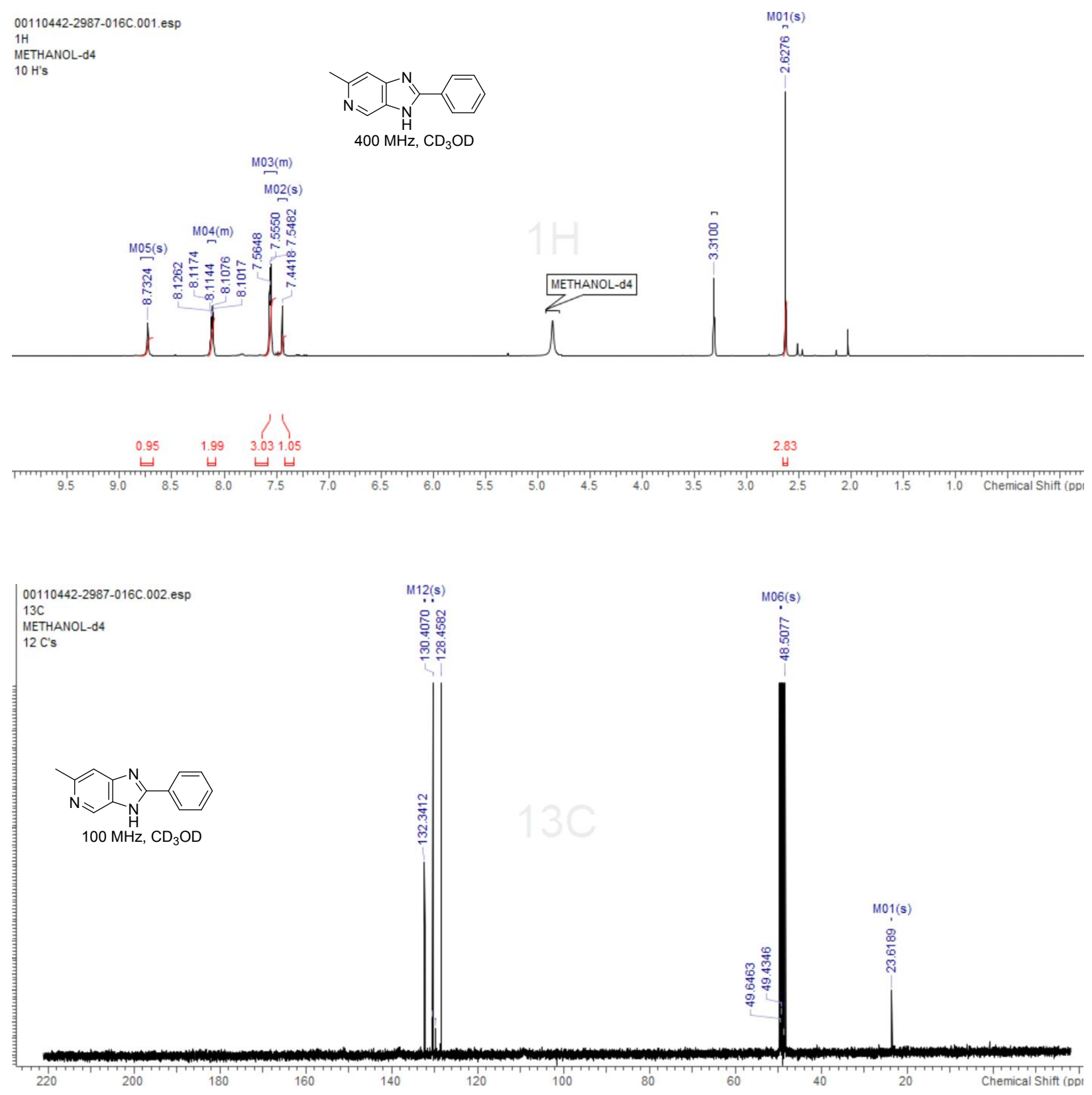
7-fluoro-2-phenyl-1H-imidazo[4,5-c]pyridine (6g)

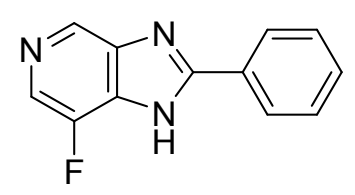

$\underline{{ }^{1} \mathbf{H} \text { NMR }}\left(400 \mathrm{MHz}, \mathrm{CD}_{3} \mathrm{OD}\right): \delta 9.04(\mathrm{~s}, 1 \mathrm{H}), 8.58(\mathrm{~d}, J=3.9 \mathrm{~Hz}, 1 \mathrm{H}), 8.29-8.26(\mathrm{~m}, 2 \mathrm{H})$, 7.68-7.63 (m, 3H) ppm.

${ }^{13} \mathrm{C} \mathrm{NMR}\left(100 \mathrm{MHz}, \mathrm{CD}_{3} \mathrm{OD}\right): \delta 162.8,159.4,149.6,133.3,133.2,130.6,129.5,129.0,127.2,127.0 \mathrm{ppm}$.

$\underline{\text { LC-MS }}$ (ESI) Calcd. for $\mathrm{C}_{12} \mathrm{H}_{8} \mathrm{FN}_{3}(\mathrm{M}+\mathrm{H}): 214.1$, Found: 214.3 .

Regioselectivity 4:1 - Assigned by ${ }^{1} \mathrm{HNMR}$. 
O0110442-2987-002A_001.esp
$1 H$
METHANOL-d4
7 H's
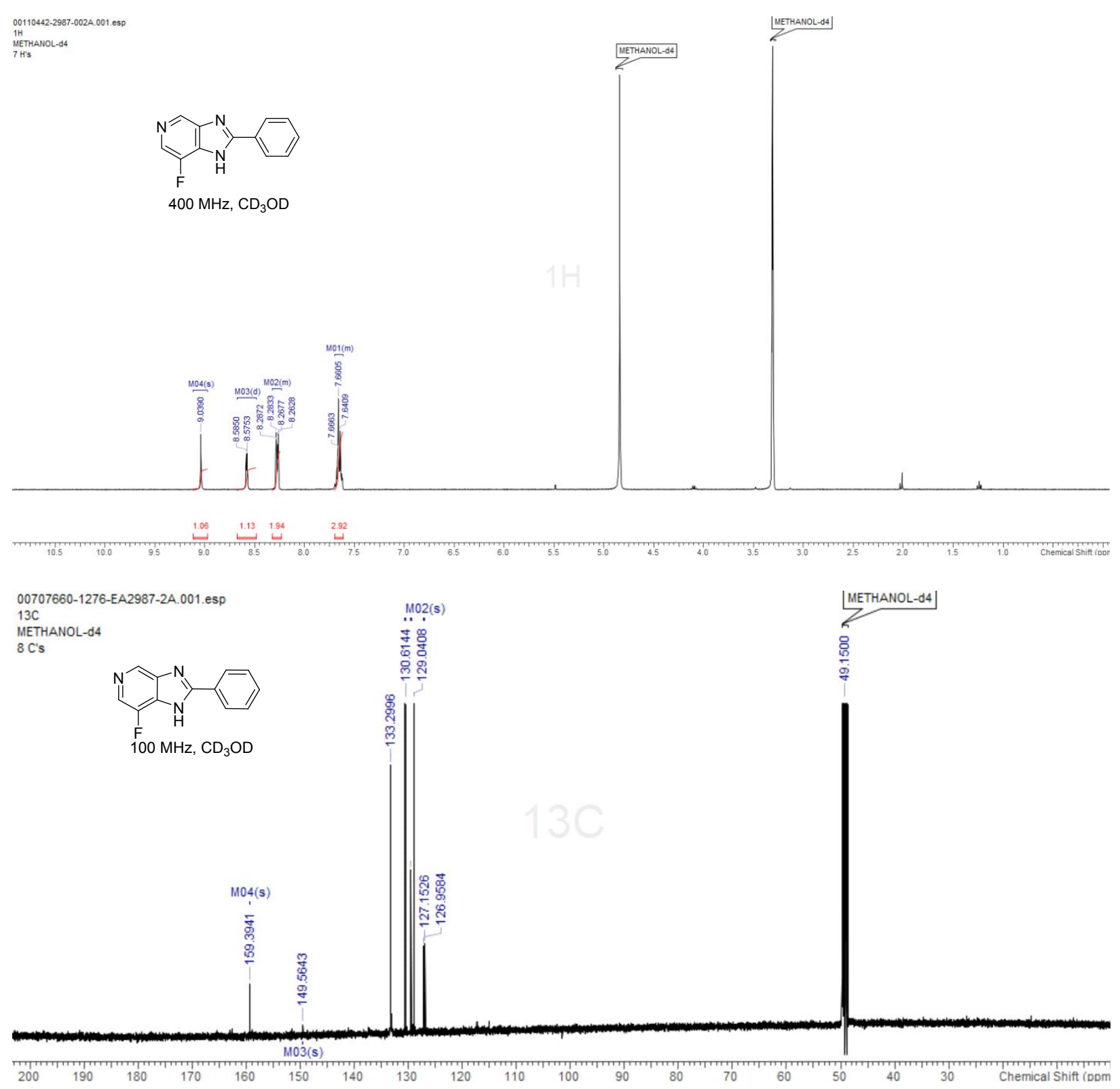

8-phenyl-9H-purine (6h) 


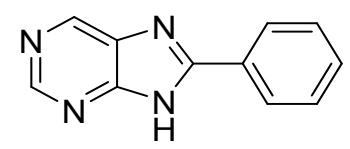

$\underline{{ }_{1} \mathbf{H ~ N M R}}\left(400 \mathrm{MHz}, \mathrm{CD}_{3} \mathrm{OD}\right): \delta 9.15$ (br s, 1H), $9.03(\mathrm{~s}, 1 \mathrm{H}), 8.25-8.22(\mathrm{~m}, 2 \mathrm{H}), 7.66-7.60$ (m, 3H) ppm.

${ }^{13}$ C NMR $\left(100 \mathrm{MHz}, \mathrm{CD}_{3} \mathrm{OD}\right): \delta 158.2,157.4,150.0,141.1,132.1,129.1,128.0,127.6,127.5 \mathrm{ppm}$.

LC-MS (ESI) Calcd. for $\mathrm{C}_{11} \mathrm{H}_{8} \mathrm{~N}_{4}(\mathrm{M}+\mathrm{H})$ : 197.1, Found: 197.3.
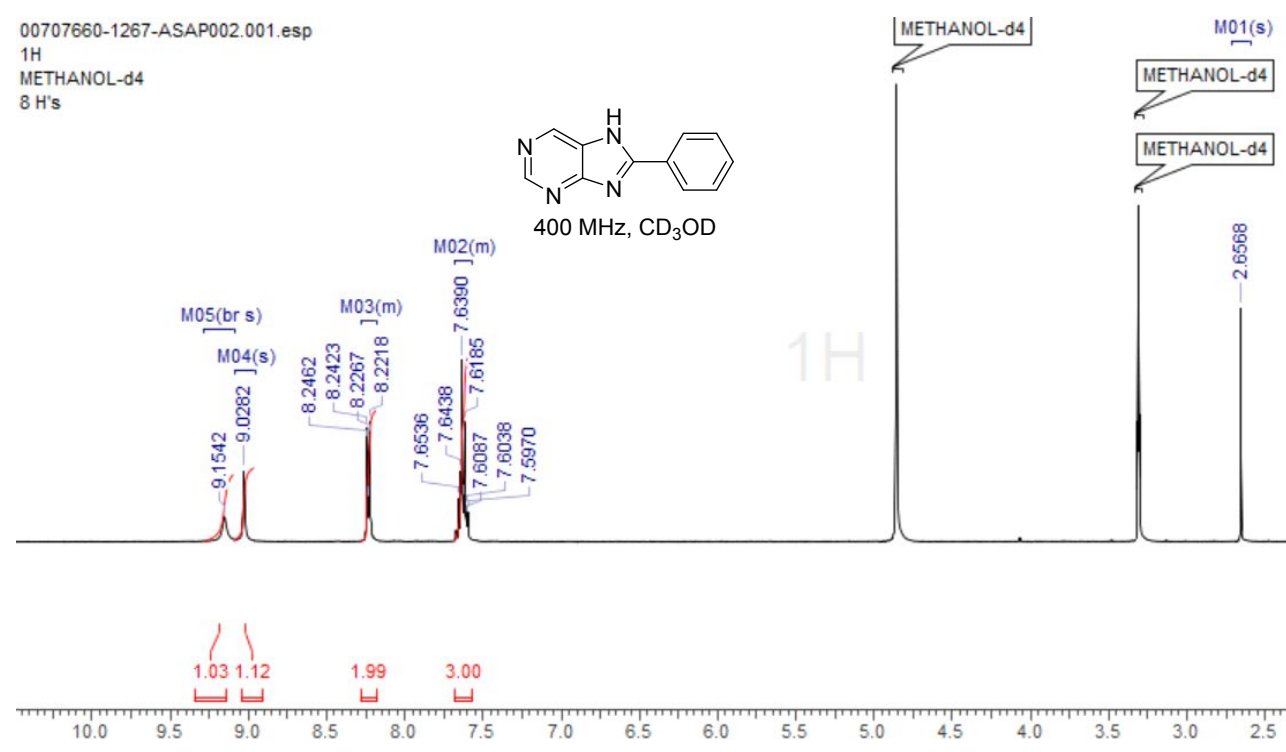


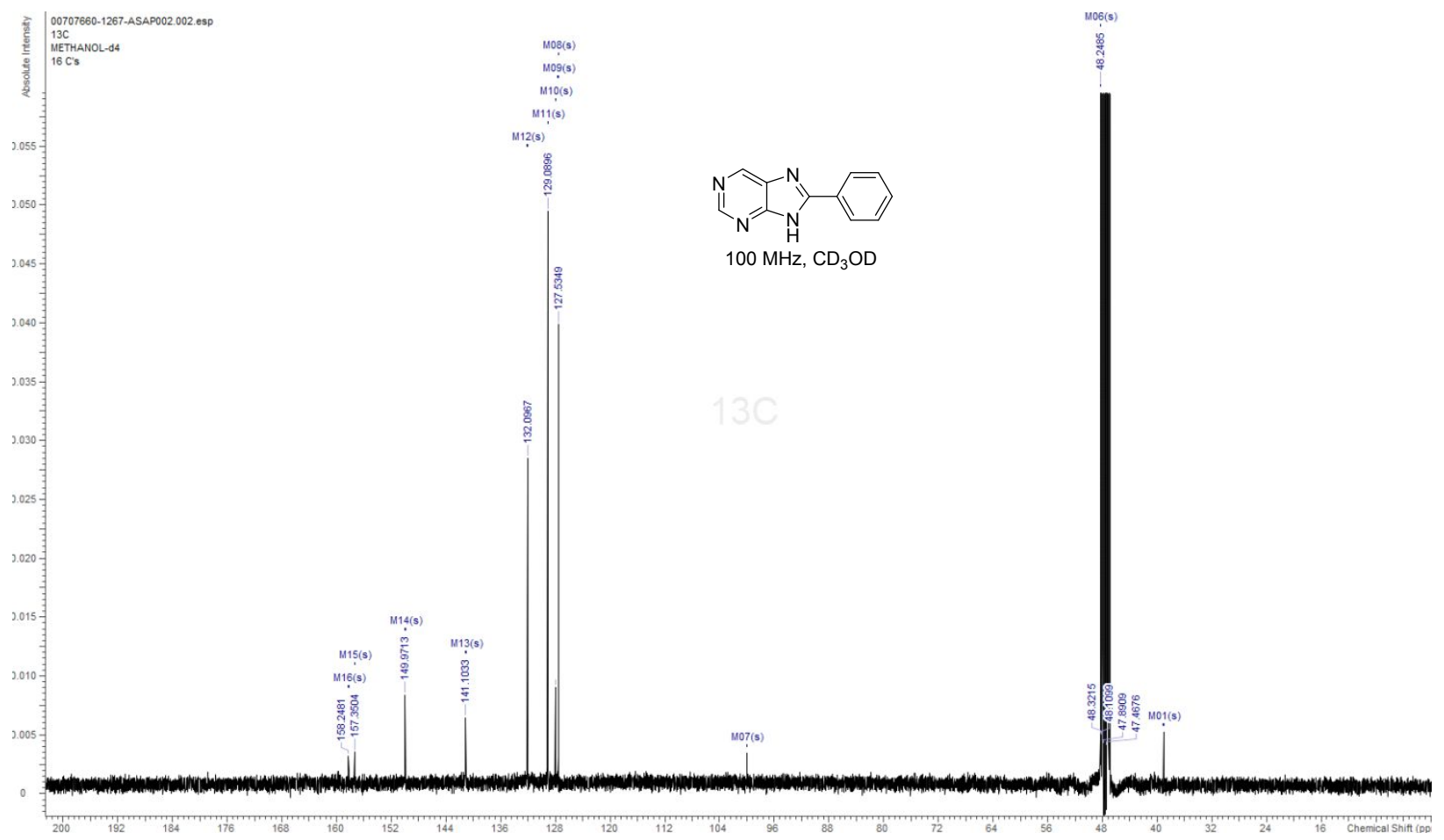

2-isopropyl-8-phenyl-9H-purine (6i)

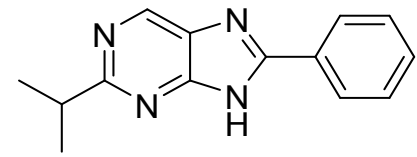

$\underline{{ }^{1} \mathbf{H} \text { NMR }}\left(400 \mathrm{MHz}, \mathrm{CD}_{3} \mathrm{OD}\right): \delta 9.15(\mathrm{~s}, 1 \mathrm{H}), 8.24-8.22(\mathrm{~m}, 2 \mathrm{H}), 7.66-7.61(\mathrm{~m}, 3 \mathrm{H}), 3.46--3.40(\mathrm{~m}, 1 \mathrm{H})$, $1.47(\mathrm{~d}, J=5.9 \mathrm{~Hz}, 6 \mathrm{H}) \mathrm{ppm}$.

${ }^{13} \mathrm{C}$ NMR $\left(100 \mathrm{MHz}, \mathrm{CD}_{3} \mathrm{OD}\right): 167.4,162.5,161.4,160.4,139.8,133.9,131.7,130.7,129.2,36.8,21.9$ ppm.

$\underline{\text { LC-MS }}$ (ESI) Calcd. for $\mathrm{C}_{14} \mathrm{H}_{14} \mathrm{~N}_{4}(\mathrm{M}+\mathrm{H}): 239.1$, Found: 239.2 . 


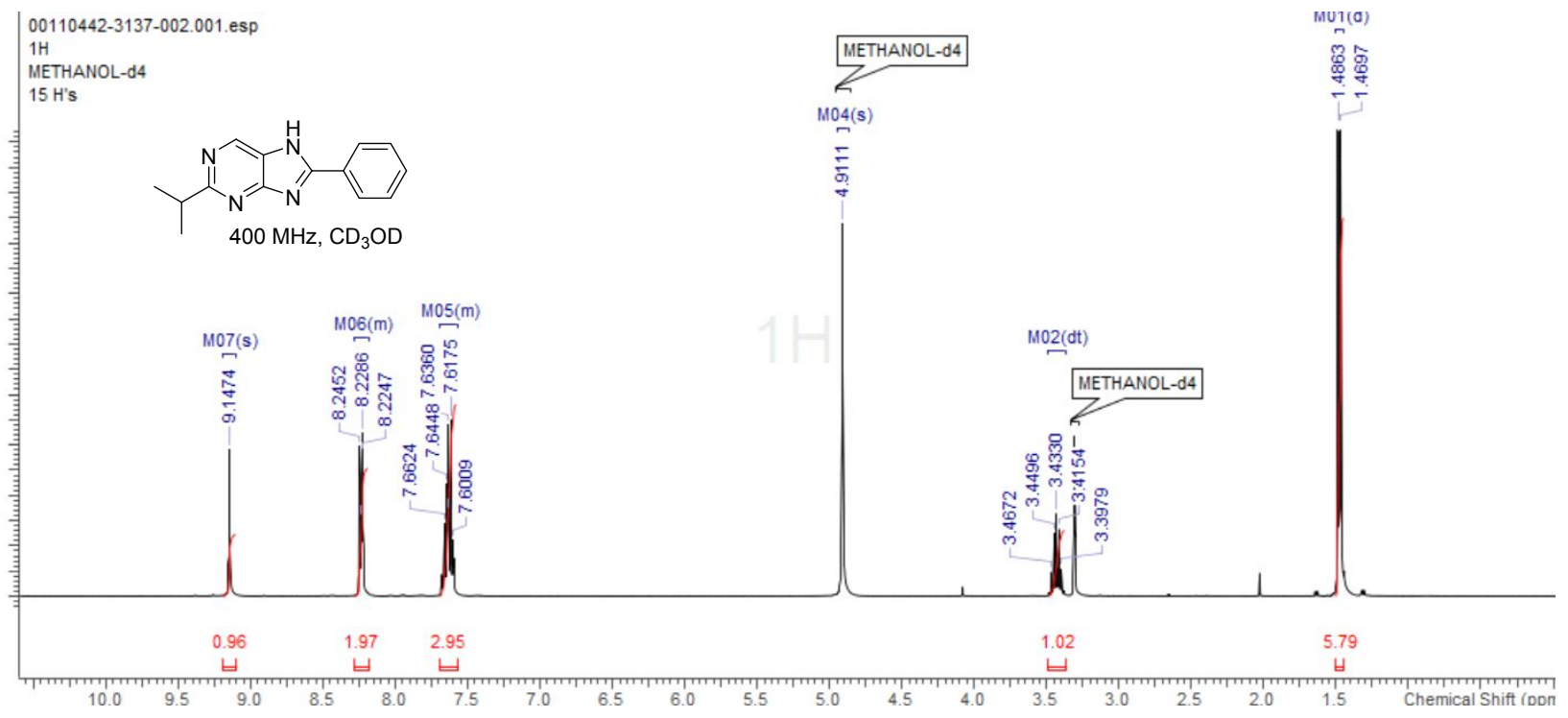




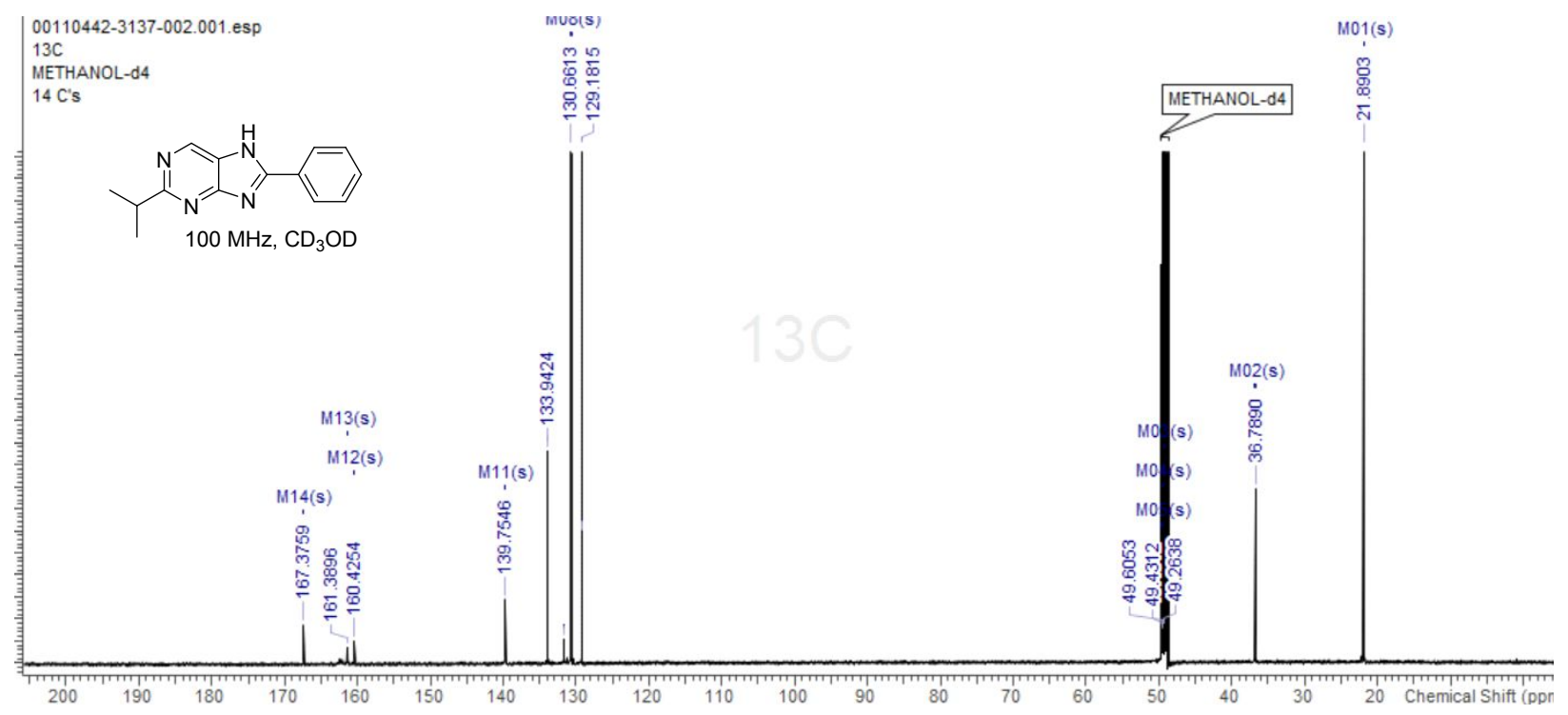

